\title{
Tachyon condensation in magnetic compactifications
}

\author{
Wilfried Buchmuller, ${ }^{a}$ Emilian Dudas ${ }^{b}$ and Yoshiyuki Tatsuta ${ }^{c, a}$ \\ ${ }^{a}$ Deutsches Elektronen-Synchrotron DESY, \\ 22607 Hamburg, Germany \\ ${ }^{b}$ CPHT, CNRS, Ecole Polytechnique, IP Paris, \\ 91128 Palaiseau, France \\ ${ }^{c}$ Scuola Normale Superiore and INFN, \\ Piazza dei Cavalieri 7, 56126 Pisa, Italy \\ E-mail: wilfried.buchmueller@desy.de, \\ emilian.dudas@cpht.polytechnique.fr, yoshiyuki.tatsuta@sns.it
}

ABSTRACT: Intersecting D-brane models and their T-dual magnetic compactifications provide an attractive framework for particle physics, allowing for chiral fermions and supersymmetry breaking. Generically, magnetic compactifications have tachyons that are usually removed by Wilson lines. However, quantum corrections prevent local minima for Wilson lines. We therefore study tachyon condensation in the simplest case, the magnetic compactification of type I string theory on a torus to eight dimensions. We find that tachyon condensation restores supersymmetry, which is broken by the magnetic flux, and we compute the Kaluza-Klein mass spectrum. The gauge group $\mathrm{SO}(32)$ is broken to USp(16). We give arguments that the vacuum reached by tachyon condensation corresponds to the unique $8 \mathrm{~d}$ superstring theory already known in the literature, with discrete $B_{a b}$ background or, in the T-dual version, the type IIB orientifold with three O7_-planes, one $\mathrm{O} 7_{+}$-plane and eight $\mathrm{D} 7$-branes coincident with the $\mathrm{O} 7_{+}$-plane. The ground state after tachyon condensation is supersymmetric and has no chiral fermions.

Keywords: D-branes, Tachyon Condensation

ArXiv EPrint: 2010.10891 


\section{Contents}

1 Introduction 1

2 Magnetic compactification to eight dimensions 2

2.1 Intersecting D8-branes 2

2.2 Effective field theory in eight dimensions 4

3 Symmetry breaking and Wilson line $\quad 8$

4 Dilute vs. singular flux 12

5 Massive spectrum for singular flux $\quad 14$

$\begin{array}{lll}6 & \text { An alternative D9-D7 brane description } & 17\end{array}$

7 The $8 \mathrm{~d}$ USp(16) superstring as magnetized type I superstring after $\begin{array}{ll}\text { tachyon condensation } & 20\end{array}$

8 Conclusions and open questions $\quad 23$

$\begin{array}{ll}\text { A Flux quantization } & 24\end{array}$

\section{Introduction}

Intersecting D-brane models and their T-dual magnetic compactifications remain an attractive framework for string theory compactifications to four dimensions with chiral fermion spectra [1]. The rich structure of D-branes and fluxes provides an intuitive way to explore the structure of string vacua with unbroken as well as broken supersymmetry $[2,3]$. In the absence of any hint for supersymmetry at the Large Hadron Collider, extensions of the Standard Model where supersymmetry is broken at a high scale are of particular interest, and magnetic string compactifications provide a theoretical framework for phenomenological models like 'split supersymmetry' [4-6] or 'split symmetries' [7].

An intriguing aspect of magnetic compactifications is the connection between fermion chirality and supersymmetry breaking [8], which occurs in compactifications of type I strings on tori and orbifolds [9-11], and correspondingly in the related intersecting Dbrane models $[10,12,13]$. In this framework compactifications have been constructed that come rather close to the Standard Model of particle physics [14-17]. A generic feature of models with broken supersymmetry is the appearance of tachyons. In all known orientifold constructions with broken supersymmetry they are removed by means of Wilson lines that correspond to flat directions in the tree-level potential. However, in a representative 
example, we recently showed that quantum corrections render these vacua unstable and that the theory is always driven to the tachyonic regime [18], which represents a serious challenge to the known models.

In principle, tachyonic instabilities are not necessarily a problem, and tachyon condensation may even lead to vacua that are phenomenologically welcome. However, the process of tachyon condensation is complicated [19-22]. In the following we therefore study the simplest case in some depth, namely type I string compactification on a magnetized torus to eight dimensions. The magnetic flux breaks the symmetry group $\mathrm{SO}(32)$ to $\mathrm{U}(16)$ and produces two multiplets of tachyons. We find that their condensation breaks $\mathrm{U}(16)$ further to $\operatorname{USp}(16)$ and restores supersymmetry, which is broken by the magnetic flux. We also compute the Kaluza-Klein mass spectrum. Remarkably, this turns out to be consistent with the unique $8 \mathrm{~d}$ superstring theory that is already known in the literature. It has gauge group USp(16) and contains a discrete $B_{a b}$ flux background. In the T-dual version, this corresponds to the type IIB orientifold with three $\mathrm{O} 7$--planes and one $\mathrm{O} 7+$-plane, with eight D7-branes required by tadpole cancellation coincident with the $\mathrm{O} 7_{+}$-plane.

The paper is organized as follows. In section 2 we briefly discuss the magnetic compactification to eight dimensions, its partition function, the tachyonic instability and the effective field theory in eight dimensions. The symmetry breaking to USp(16) is explained in section 3. Moreover, a Wilson line is considered that breaks $\mathrm{SO}(32)$ to $\mathrm{U}(16)$ rather than USp(16), leaving supersymmetry unbroken. Tachyon condensation is discussed in section 4 and shown to lead to singular localized flux. Supersymmetry is unbroken and there are no chiral fermions. As shown in section 5, the mass spectrum corresponds to a free theory with twisted boundary conditions. In section 6 a brane description of the vacuum after tachyon condensation is described, which is shown to correspond to the unique $8 \mathrm{~d}$ USp(16) in section 7 . In the appendix, for the convenience of the reader, we recall flux quantization on the torus in the symmetric gauge.

\section{Magnetic compactification to eight dimensions}

\subsection{Intersecting D8-branes}

Let us consider the simplest system in which tachyon condensation occurs: intersecting D8-branes in type IIA string theory compactified on a toroidal orientifold with two compact dimensions, a setup very similar to the magnetic compactifications to six dimensions considered in [10]. Here the 10d theory is compactified on a torus to $8 \mathrm{~d}$ Minkowski space with real coordinates $x_{0}, \ldots, x_{3}$ and complex coordinates $z_{i}=\left(x_{2+2 i}+i x_{3+2 i}\right) / 2, i=1,2$. The complex torus coordinate is $z \equiv z_{3}$, with the identifications $z \sim z+\pi R, z \sim z+i \pi R^{\prime}$. An orientifold is obtained by dividing out the discrete symmetry $\Omega \mathcal{R}(-1)^{F_{L}}$, where $\Omega$ is worldsheet parity, $F_{L}$ is left-moving fermion number, and $\mathcal{R}$ is the symmetry of $T^{2}$ under the reflection $z \rightarrow \bar{z}$. The orientifold has two O8-planes along 8d Minkowski space, and the $x_{8}$-direction is invariant under $\mathcal{R}$. The orientifold planes are localized at the fixed points $x_{9}=0, \pi R^{\prime}$. Each orientifold plane has RR charge $Q_{\mathrm{O} 8}=-16$ in units of a D8-brane charge. A stack of $N$ D8-branes requires a stack of $N$ mirror D8-branes to satisfy the reflection symmetry $\mathcal{R}$ of the compact space. The branes are wrapped around the 1-cycles 
$[a]$ and $[b]$ of the 2-torus with wrapping numbers $n$ and $m$, corresponding to the homology class $[\Pi]=n[a]+m[b]$. The homology class $[\Pi]^{\prime}$ of the mirror brane is obtained from $[\Pi]$ by replacing $m$ by $-m$. The stack of $N$ branes carries the gauge symmetry $\mathrm{U}(N)$, and the $\mathrm{RR}$ tadpole cancellation condition reads

$$
N\left([\Pi]+[\Pi]^{\prime}\right)-16\left[\Pi_{\mathrm{O} 8}\right]=0 .
$$

For simplicity we restrict ourselves to the case $n=1$. With $\left[\Pi_{\mathrm{O} 8}\right]=2[a]$, this implies $N=16$.

The stack of branes leads to a tower of $8 \mathrm{~d} \mathcal{N}=1$ supermultiplets in the adjoint representation $\mathbf{2 5 6}$ of the gauge group $\mathrm{U}(16)$. Branes and mirror branes intersect at an angle determined by the wrapping numbers. The wrapping numbers we consider here are $(n, m)=(1, k)$ for the D8-branes and $(1,-k)$ for their mirrors. At their intersection a massless chiral $8 \mathrm{~d} \mathcal{N}=1$ fermion in the antisymmetric representation $\mathbf{1 2 0}$ of $\mathrm{U}(16)$ is localized, together with massive fermions, vectors, scalars and tachyons, all in the antisymmetric representation. Their masses depend on the intersection angle $\theta$ that breaks supersymmetry, and their multiplicity is given by the intersection number $I$ that is related to the wrapping number $k$ by [10]

$$
I=2 k \text {. }
$$

The scalar masses of the antisymmetric tensors depend on the angle with the orientifold plane. We restrict ourselves to small angles with respect to the orientifold plane,

$$
\tan \theta=k \rho \simeq \theta, \quad \rho=\frac{R_{2}}{R_{1}},
$$

where $R_{1}$ and $R_{2}$ are the two radii of the torus. In the T-dual picture small angles correspond to large areas of the dual torus so that we are able to use a field theory approximation to string partition functions.

At the intersection of the two stacks of branes and mirror branes one then has three light scalars with masses [13]

$$
4 \pi \alpha^{\prime} M_{1}^{2}=-2|\theta|, \quad 4 \pi \alpha^{\prime} M_{2}^{2}=2|\theta|, \quad 4 \pi \alpha^{\prime} M_{3}^{2}=2|\theta|,
$$

where $-\pi / 2 \leq \theta \leq \pi / 2$. Clearly, the model has a tachyon with negative mass squared $M_{1}^{2}=-k \rho /\left(2 \pi \alpha^{\prime}\right)$.

The intersecting D-brane model described above is T-dual to a type I compactification on a magnetized dual rectangular torus $T^{2}$ with the identifications

$$
z \sim z+\pi R_{1}, \quad z \sim z+i \pi \alpha^{\prime} / R_{2} .
$$

The area of the dual torus is $4 \pi^{2} \alpha^{\prime} / \rho$, and the angle $\theta$ between the stack of branes and the orientifold plane is related to a magnetic flux density $f$ on the torus $T^{2}$ [12],

$$
\tan \theta=2 \pi \alpha^{\prime} g f
$$

where $g$ is a gauge coupling. Using eq. (2.3), this implies the quantization condition for the flux density $f$,

$$
\frac{g}{2 \pi} \int_{T^{2}} f=k
$$


The mass spectrum of the intersection D8-branes is encoded in the magnetic deformation of the open string part of the type I partition function. ${ }^{1}$ This is the sum of annulus amplitude $\mathcal{A}$ and Moebius amplitude $\mathcal{M}$, which can be compactly expressed in terms of standard modular functions and $\mathrm{SO}(8)$ characters [2],

$$
\begin{aligned}
& \mathcal{A}=\int_{0}^{\infty} \frac{d \tau_{2}}{\tau_{2}^{5}}\left\{N \bar{N} \frac{\left(V_{8}-S_{8}\right)(0 \mid \tau)}{\eta^{8}}+\right. \\
&\left.2 k\left[\frac{N^{2}}{2}\left(V_{8}-S_{8}\right)(2 \epsilon \tau \mid \tau)+\frac{\bar{N}^{2}}{2}\left(V_{8}-S_{8}\right)(-2 \epsilon \tau \mid \tau)\right] \frac{i}{\theta_{1}(2 \epsilon \tau \mid \tau) \eta^{5}}\right\} \\
& \mathcal{M}=-k \int_{0}^{\infty} \frac{d \tau_{2}}{\tau_{2}^{5}}\left[N\left(\hat{V}_{8}-\hat{S}_{8}\right)(2 \epsilon \tau \mid \tau)+\bar{N}\left(\hat{V}_{8}-\hat{S}_{8}\right)(-2 \epsilon \tau \mid \tau)\right] \frac{i}{\hat{\theta}_{1}(2 \epsilon \tau \mid \tau) \hat{\eta}^{5}}
\end{aligned}
$$

where for the annulus amplitude $\tau=i \tau_{2} / 2$, whereas for the Moebius amplitude $\tau=i \tau_{2} / 2+1 / 2$, and $N=\bar{N}=16$. The hatted characters were defined originally in refs. [23, 24]. The partition function contains the sum over string oscillator modes and Landau-level modes, whose masses are inversely proportional to the area of the torus. Their contribution to the one-loop effective potential $V=-\mathcal{A}$ is obtained by considering the $\tau_{2} \rightarrow \infty$ limit of the modular functions. Changing variables from $\tau_{2}$ to the Schwinger proper time $t=\pi \alpha^{\prime} \tau_{2}$, the result for the second term in eq. (2.8) can be written as [18],

$$
V=\int_{0}^{\infty} d t V(t) \propto \int_{0}^{\infty} \frac{d t}{t^{5}} \frac{\sinh ^{4}\left(\frac{g f t}{2}\right)}{\sinh (g f t)} .
$$

The integral has an infrared divergence at $t \rightarrow \infty$, where the integrand behaves as

$$
V(t) \propto \frac{1}{t^{5}} e^{g f t}
$$

This is the effect of the tachyon with negative mass square $M_{T}^{2}=-g f$.

\subsection{Effective field theory in eight dimensions}

Neglecting oscillator modes, the open string sector of the type I superstring becomes the 10d Super-Yang-Mills action with gauge group SO(32). In order to obtain the 8d effective field theory it is convenient to start from the 10d Super-Yang-Mills action expressed in terms of $4 \mathrm{~d} \mathcal{N}=1$ vector superfield $V$ and chiral superfields $\phi[25,26]$,

$$
\begin{aligned}
& S_{10}=\int d^{10} x\left\{\frac{1}{\tilde{k}} \int d^{2} \theta \operatorname{Tr}\right. {\left[\frac{1}{4} \hat{W} \hat{W}+\frac{1}{2} \varepsilon_{i j k} \hat{\phi}^{i}\left(\partial_{j} \hat{\phi}^{k}+\frac{g}{3 \sqrt{2}}\left[\hat{\phi}^{j}, \hat{\phi}^{k}\right]\right)\right]+\text { h.c. } } \\
&+\frac{1}{\tilde{k}} \int d^{4} \theta \operatorname{Tr}\left[\hat{\phi}^{i \dagger} \hat{\phi}^{i}+\sqrt{2}\left(\partial_{i} \hat{\phi}^{i \dagger}+\bar{\partial}_{i} \hat{\phi}^{i}\right) \hat{V}-g\left[\hat{\phi}^{i \dagger}, \hat{\phi}^{i}\right] \hat{V}\right. \\
&\left.\left.+\left(\partial_{i} \hat{V}+\frac{g}{\sqrt{2}}\left[\hat{V}, \hat{\phi}^{i}\right]\right)^{\dagger}\left(\partial_{i} \hat{V}+\frac{g}{\sqrt{2}}\left[\hat{\phi}^{i}, \hat{V}\right]\right)\right]\right\} .
\end{aligned}
$$

\footnotetext{
${ }^{1}$ For simplicity, we drop the overall factor $1 /\left(2\left(4 \pi^{2} \alpha^{\prime}\right)^{4}\right)$.
} 
Here $\hat{\phi}^{i}$ are chiral superfields, $i, j, k=1,2,3, \hat{V}$ is a vector superfield and $\hat{W}$ is the corresponding field strength. ${ }^{2}$ The hat denotes $\mathrm{SO}(32)$ matrices. 10d Minkowski space is described by real coordinates $x_{0}, \ldots, x_{3}$, complex coordinates $z_{i}=\left(x_{2+2 i}+i x_{3+2 i}\right) / 2$, $i=1,2$ and $z_{3} \equiv z$; complex derivatives are defined as $\partial_{i}=\partial / \partial z_{i}, \bar{\partial}_{i}=\partial / \partial \bar{z}_{i}$. For simplicity, we consider compactification to $8 \mathrm{~d}$ Minkowski space on a square torus $T^{2}$ with the identifications $z \sim z+\pi R, z \sim z+i \pi R$.

It is convenient to write the $\mathrm{SO}(32)$ matrix valued fields in block form, ${ }^{3}$ such that the blocks transform with respect to the $\mathrm{U}(16)$ subgroup as adjoint or antisymmetric representation, respectively,

$$
\begin{aligned}
\hat{V} & =\left(\begin{array}{cc}
V & \sqrt{2} V^{+} \\
\sqrt{2} V^{-} & -V^{T}
\end{array}\right), \quad V=V^{\dagger}, V^{-}=V^{+\dagger}, \\
\hat{\phi}^{i} & =\left(\begin{array}{cc}
\phi^{i} & \sqrt{2} \phi^{i+} \\
\sqrt{2} \phi^{i-} & -\phi^{i T}
\end{array}\right) .
\end{aligned}
$$

In terms of these fields ${ }^{4}$ the 10d Lagrangian reads

$$
\begin{aligned}
& \mathcal{L}_{10}=\int d^{2} \theta \operatorname{tr}\left[\frac{1}{4} W_{V} W_{V}+\frac{1}{2} W^{+} W^{-}+\varepsilon_{i j k}\left(\frac{1}{2} \phi^{i} \partial_{j} \phi^{k}\right.\right. \\
&+\left.\left.\phi^{i+} \partial_{j} \phi^{k-}+\frac{g}{3 \sqrt{2}} \phi^{i} \phi^{j} \phi^{k}-\sqrt{2} g \phi^{i+} \phi^{k-} \phi^{j}\right)\right]+ \text { h.c. } \\
&+\int d^{4} \theta \operatorname{tr}\left[\bar{\phi}^{i} \phi^{i}+\bar{\phi}^{i+} \phi^{i+}+\bar{\phi}^{i-} \phi^{i-}\right. \\
&+\left(\sqrt{2}\left(\partial_{i} \bar{\phi}^{i}+\bar{\partial}_{i} \phi^{i}\right)-g\left[\bar{\phi}^{i}, \phi^{i}\right]-2 g\left(\bar{\phi}^{i-} \phi^{i-}-\phi^{i+} \bar{\phi}^{i+}\right)\right) V \\
&+\left(\sqrt{2}\left(\partial_{i} \bar{\phi}^{i-}+\bar{\partial}_{i} \phi^{i+}\right)-2 g\left(\bar{\phi}^{i} \phi^{i+}-\phi^{i} \bar{\phi}^{i-}\right)\right) V^{-} \\
&+\left(\sqrt{2}\left(\partial_{i} \bar{\phi}^{i+}+\bar{\partial}_{i} \phi^{i-}\right)-2 g\left(\bar{\phi}^{i+} \phi^{i}-\phi^{i-} \bar{\phi}^{i}\right)\right) V^{+} \\
&+\left(\bar{\partial}_{i} V+\frac{g}{\sqrt{2}}\left[V, \bar{\phi}^{i}\right]+\sqrt{2} g\left(V^{+} \bar{\phi}^{i+}-\bar{\phi}^{i-} V^{-}\right)\right) \\
& \times\left(\partial_{i} V+\frac{g}{\sqrt{2}}\left[\phi^{i}, V\right]+\sqrt{2} g\left(\phi^{i+} V^{-}-V^{+} \phi^{i-}\right)\right) \\
&+\left(\bar{\partial}_{i} V^{+}-\frac{g}{\sqrt{2}}\left(\bar{\phi}^{i} V^{+}+V^{+} \bar{\phi}^{i T}-V \bar{\phi}^{i-}-\bar{\phi}^{i-} V^{T}\right)\right) \\
& \times\left(\partial_{i} V^{-}-\frac{g}{\sqrt{2}}\left(V^{-} \phi^{i}+\phi^{i T} V^{-}-\phi^{i-} V-V^{T} \phi^{i-}\right)\right) \\
&+\left(\bar{\partial}_{i} V^{-}+\frac{g}{\sqrt{2}}\left(V^{-} \bar{\phi}^{i}+\bar{\phi}^{i T} V^{-}-\bar{\phi}^{i+} V-V^{T} \bar{\phi}^{i+}\right)\right) \\
&\left.\times\left(\partial_{i} V^{+}+\frac{g}{\sqrt{2}}\left(\phi^{i} V^{+}+V^{+} \phi^{i T}-V \phi^{i+}-\phi^{i+} V^{T}\right)\right)\right] .
\end{aligned}
$$

\footnotetext{
${ }^{2}$ We use the conventions of [27], and we have dropped the WZW term that vanishes in WZ gauge, $V^{3}=0$. Our trace convention is $\operatorname{Tr}\left[\hat{T}_{a} \hat{T}_{b}\right]=\tilde{k} \delta_{a b}, \tilde{k}=2$.

${ }^{3}$ For a recent discussion, see [18].

${ }^{4}$ For fields we use the notation $V^{\dagger}=\bar{V}, V^{+\dagger}=\bar{V}^{+}=V_{-}, \phi^{\dagger}=\bar{\phi}$ etc. $\operatorname{tr}$ denotes the trace of $\mathrm{U}(16)$ matrices with the convention $\operatorname{tr}\left[T^{a} T^{b}\right]=\delta_{a b}$, see appendix A of [18].
} 
Consider now a background field with constant magnetic flux $\left(\phi^{3} \equiv \phi\right)$, which breaks $\mathrm{SO}(32)$ to $\mathrm{U}(16)$,

$$
\langle\phi\rangle=\frac{1}{\sqrt{2}} f \bar{z} \mathbb{1},
$$

and which is a solution of the equation of motion,

$$
\partial(\partial\langle\bar{\phi}\rangle+\bar{\partial}\langle\phi\rangle)=0
$$

The background field leads to Landau-level type mass terms for charged fields. They are most easily obtained by combining the magnetic flux with derivatives to annihilation and creation operators [28],

$$
\begin{array}{ll}
\partial-g f \bar{z}=-i \sqrt{2 g f} a_{-}^{\dagger}, & \bar{\partial}+g f z=-i \sqrt{2 g f} a_{-}, \\
\bar{\partial}-g f z=-i \sqrt{2 g f} a_{+}^{\dagger}, & \partial+g f \bar{z}=-i \sqrt{2 g f} a_{+},
\end{array}
$$

which satisfy the commutation relations

$$
\left[a_{ \pm}, a_{ \pm}^{\dagger}\right]=1, \quad\left[a_{ \pm}, a_{\mp}\right]=0, \quad\left[a_{ \pm}, a_{\mp}^{\dagger}\right]=0 .
$$

The relevant linear and bilinear terms of the Lagrangian $(2.14)$ are $\left(i, j=1,2, \phi^{3 \pm}=\phi^{ \pm}\right)$

$$
\begin{aligned}
\mathcal{L}_{10} \supset \operatorname{tr}\left[\int d^{2} \theta i \sqrt{2 g f} \varepsilon_{i j} \phi^{i+} a_{-}^{\dagger} \phi^{j-}+\right.\text { h.c. } \\
+\int d^{4} \theta\left(2\left(f-g\left(\bar{\phi}^{i-} \phi^{i-}+\bar{\phi}^{-} \phi^{-}-\phi^{i+} \bar{\phi}^{i+}-\phi^{+} \bar{\phi}^{+}\right)\right) V\right. \\
-2 i \sqrt{g f}\left(\left(a_{-} \phi^{-}+a_{-}^{\dagger} \bar{\phi}^{+}\right) V^{+}+\left(a_{+} \bar{\phi}^{-}+a_{+}^{\dagger} \phi^{+}\right) V^{-}\right) \\
\left.\left.-2 g f\left(a_{+}^{\dagger} V^{+} a_{-}^{\dagger} V^{-}+a_{-} V^{-} a_{+} V^{+}\right)\right)\right] .
\end{aligned}
$$

Expanding the charged fields in the standard harmonic oscillator mode functions,

$$
\begin{aligned}
& \phi^{+}=\sum_{n, j} \phi_{n, j}^{+} \xi_{n, j}, \quad \phi^{-}=\sum_{n, j} \phi_{n, j}^{-} \bar{\xi}_{n, j}, \quad \bar{\phi}^{+}=\sum_{n, j} \bar{\phi}_{n, j}^{+} \bar{\xi}_{n, j}, \\
& \bar{\phi}^{-}=\sum_{n, j} \bar{\phi}_{n, j}^{-} \xi_{n, j}, \quad V^{+}=\sum_{n, j} V_{n, j}^{+} \xi_{n, j}, \quad V^{-}=\sum_{n, j} V_{n, j}^{-} \bar{\xi}_{n, j},
\end{aligned}
$$

which satisfy

$$
\begin{array}{ll}
a_{+} \xi_{n, j}=i \sqrt{n} \xi_{n-1, j}, & a_{+}^{\dagger} \xi_{n, j}=-i \sqrt{n+1} \xi_{n+1, j}, \\
a_{-} \bar{\xi}_{n, j}=i \sqrt{n} \bar{\xi}_{n-1, j}, & a_{-}^{\dagger} \bar{\xi}_{n, j}=-i \sqrt{n+1} \bar{\xi}_{n+1, j},
\end{array}
$$


keeping only the zero mode of $V$, and integrating over the torus coordinates, one obtains ${ }^{5}$

$$
\begin{aligned}
\mathcal{L}_{8} \supset \operatorname{tr}\left[\int d^{2} \theta \sum_{n} \sqrt{2 g f(n+1)} \varepsilon_{i j} \phi_{n+1}^{i+} \phi_{n}^{j-}+\right.\text { h.c. } \\
+\int d^{4} \theta\left(\left(2 f-2 g \sum_{n}\left(\bar{\phi}_{n}^{i-} \phi_{n}^{i-}-\phi_{n}^{i+} \bar{\phi}_{n}^{i+}\right)\right) V\right. \\
+2 \sqrt{g f}\left(\left(\sqrt{n+1} \phi_{n+1}^{-}-\sqrt{n} \bar{\phi}_{n-1}^{+}\right) V_{n}^{+}+\text {h.c. }\right) \\
\left.\left.-2 g f(2 n+1) V_{n}^{+} V_{n}^{-}\right)\right] .
\end{aligned}
$$

Magnetic flux breaks supersymmetry. To find the mass spectrum one therefore has to expand superfields in components, ${ }^{6}$

$$
\phi=(\phi, \psi, F), \quad V=\left(A_{\mu}, \lambda, D\right) .
$$

The mixing term between chiral and vector superfields contains D-terms and derivative couplings between Goldstone bosons and vector fields,

$$
\begin{aligned}
\sqrt{2} \int d^{4} \theta & \left(\sqrt{n} \bar{\phi}_{n-1}^{+}-\sqrt{n+1} \phi_{n+1}^{-}\right) V_{n}^{+} \\
= & \frac{1}{\sqrt{2}}\left(\sqrt{n} \bar{\phi}_{n-1}^{+}-\sqrt{n+1} \bar{\phi}_{n+1}^{-}\right) D^{+}-i \sqrt{2 n+1} \partial_{\mu} \Pi_{n}^{-} A_{n}^{+\mu} .
\end{aligned}
$$

Here the Goldstone fields $\Pi^{-}$and the orthogonal complex scalars $\Sigma^{-}$, formed from the complex scalars $\bar{\phi}^{+}$and $\phi^{-}$, are given by

$$
\begin{aligned}
& \Pi_{n}^{-}=\frac{1}{\sqrt{2(2 n+1)}}\left(\sqrt{n} \bar{\phi}_{n-1}^{+}+\sqrt{n+1} \phi_{n+1}^{-}\right), \\
& \Sigma_{n}^{-}=\frac{1}{\sqrt{2(2 n+3)}}\left(\sqrt{n+2} \bar{\phi}_{n}^{+}-\sqrt{n+1} \phi_{n+2}^{-}\right) .
\end{aligned}
$$

The vector bosons of the tower of Landau levels acquire their mass by the Stueckelberg mechanism, and a shift of the vector bosons,

$$
A_{n}^{-\mu} \rightarrow A_{n}^{-\mu}+\frac{i}{\sqrt{g f(2 n+1)}} \partial_{\mu} \Pi_{n}^{-},
$$

cancels the mixings with the Goldstone bosons as well as the kinetic terms of the Goldstone bosons. Finally, eliminating all F- and D-terms via their equations of motion, one obtains the bosonic mass terms $(i=1,2)$

$$
\begin{aligned}
\mathcal{L}_{8}^{b}=-g f \operatorname{tr}[ & \sum_{n}(2 n+1)\left(A_{n \mu}^{+} A_{n}^{-\mu}+\left|\phi_{n}^{i+}\right|^{2}+\left|\phi_{n}^{i-}\right|^{2}\right) \\
& \left.-\left|\phi_{0}^{-}\right|^{2}+\sum_{n}(2 n+3)\left|\Sigma_{n}^{-}\right|^{2}\right] .
\end{aligned}
$$

\footnotetext{
${ }^{5}$ In the following we drop the index $j$ that labels the ground state degeneracy. The multiplicity of states is given by $q g /(2 \pi) \int_{T^{2}} f=q k$ where $q$ is the charge of the field. Since the charge of the antisymmetric tensor is $q=2$, the multiplicity is $2 k$ for $k$ flux quanta.

${ }^{6}$ We use the same symbol for a chiral superfield and its scalar component.
} 
The structure of this result is easily understood. The fields $\phi_{n}^{i \pm}$ do not mix with the vector bosons, and combining $\mathrm{F}$ - and D-term contributions, one finds that they get the same mass as the vector bosons. The fields $\phi_{n}^{ \pm}$contain the Goldstone bosons for the tower of vector bosons $A_{n}^{ \pm \mu}$ and an orthogonal tower of fields $\Sigma_{n}^{-}$. Since the vectors mix with different levels of the complex scalars, the fields $\Sigma_{n}^{-}$are not mass degenerate with $\phi_{n}^{i \pm}$. Moreover, the scalar $\phi_{0}^{-}$is a tachyon. ${ }^{7}$

The fermion masses are easily read off from (2.22). Denoting the Weyl fermions contained in the superfields $\phi^{i \pm}, \phi^{ \pm}$and $V^{+}=\bar{V}^{-}$by $\psi^{i \pm}, \psi^{ \pm}$and $\lambda^{ \pm}$, respectively, one obtains

$$
\mathcal{L}_{8}^{f} \supset \sqrt{g f} \sum_{n} \sqrt{(n+1)} \operatorname{tr}\left[\varepsilon_{i j} \psi_{n+1}^{i+} \psi_{n}^{j-}+i \psi_{n+1}^{-} \lambda_{n}^{+}+i \psi_{n}^{+} \lambda_{n+1}^{-}\right]+\text {h.c. }
$$

Again, the mass terms couple different levels of the Landau towers, and the four $4 \mathrm{~d}$ Weyl fermions $\psi_{0}^{1+}, \psi_{0}^{2+}, \bar{\psi}_{0}^{-}$and $\bar{\lambda}_{0}^{-}$form one $8 \mathrm{~d}$ Weyl spinor with charge +1 . All fields are antisymmetric tensors of $\mathrm{U}(16)$ and have a multiplicity $2 k$ for $k$ flux quanta.

The negative mass squared of $\phi_{0}^{-}$in eq. (2.27) suggests that this mode will grow exponentially with time after a small perturbation around the unstable extremum $\phi_{0}^{-}=0$. At present it is an open question what the final state after tachyon condensation is like. Of particular interest are supersymmetric vacua which can be analyzed by means of auxiliary fields, allowing for background flux as well as Wilson lines. From eq. (2.14) one obtains

$$
\begin{aligned}
-\bar{F}^{i} & =\varepsilon_{i j k}\left(\partial_{j} \phi^{k}+\frac{g}{\sqrt{2}} \phi^{j} \phi^{k}-\sqrt{2} g \phi^{j+} \phi^{k-}\right) \\
-\bar{F}^{i+} & =\varepsilon_{i j k}\left(\partial_{j} \phi^{k-}-\sqrt{2} g \phi^{j-} \phi^{k}\right) \\
-\bar{F}^{i-} & =\varepsilon_{i j k}\left(\partial_{j} \phi^{k+}-\sqrt{2} g \phi^{j+} \phi^{k}\right) \\
-2 D & =\sqrt{2}\left(\partial_{i} \bar{\phi}^{i}+\bar{\partial}_{i} \phi^{i}\right)-g\left[\bar{\phi}^{i}, \phi^{i}\right]-2 g\left(\bar{\phi}^{i-} \phi^{i-}-\phi^{i+} \bar{\phi}^{i+}\right), \\
-D^{+} & =\sqrt{2}\left(\partial_{i} \bar{\phi}^{i-}+\bar{\partial}_{i} \phi^{i+}\right)-2 g\left(\bar{\phi}^{i} \phi^{i+}-\phi^{i} \bar{\phi}^{i-}\right), \\
-D^{-} & =\sqrt{2}\left(\partial_{i} \bar{\phi}^{i+}+\bar{\partial}_{i} \phi^{i-}\right)-2 g\left(\bar{\phi}^{i+} \phi^{i}-\phi^{i-} \bar{\phi}^{i}\right) .
\end{aligned}
$$

In a supersymmetric vacuum all auxiliary fields vanish, and vacuum field configurations can be found by solving a system of first-order differential equations.

\section{Symmetry breaking and Wilson line}

The condensation of the antisymmetric tensor $\phi_{0}^{-}$breaks U(16) to USp(16) [29]. To understand this, it is convenient to consider the following decomposition of the $\mathrm{SO}(32)$ generators. The generators of the unitary subgroup $\mathrm{U}(16)$ are hermitian matrices $S+i A$ and those of the coset $\mathrm{SO}(32) / \mathrm{U}(16)$ are complex antisymmetric matrices $A_{1}+i A_{2}$, respectively. Here $S$ denotes real $16 \times 16$ matrices, and $A, A_{1,2}$ are antisymmetric real matrices. They can be

\footnotetext{
${ }^{7}$ In [18] the case with magnetic flux in two tori was considered. The mixing pattern is then more complicated, and by tuning the fluxes one can obtain two massless scalars instead of a tachyon [13]. The masses in eq. (2.27) refer to mass eigenstates, contrary to the mass levels in Bachas' string mass formula [8]. There the longitudinal components of the vectors and the tachyon would be counted as a tower of scalars with masses $g f(2 n-1), n \geq 0$.
} 
expressed as products of Pauli matrices and $8 \times 8$ symmetric $\left(S^{\prime}\right)$ and antisymmetric $\left(A^{\prime}\right)$ matrices:

$$
\begin{aligned}
& S: \quad \sigma_{0} \otimes S^{\prime}, \quad \sigma_{1} \otimes S^{\prime}, \quad i \sigma_{2} \otimes A^{\prime}, \quad \sigma_{3} \otimes S^{\prime}, \\
& A: \quad \sigma_{0} \otimes A^{\prime}, \quad \sigma_{1} \otimes A^{\prime}, \quad i \sigma_{2} \otimes S^{\prime}, \quad \sigma_{3} \otimes A^{\prime} .
\end{aligned}
$$

Tachyon condensation removes the tachyon if the condensate cancels the flux contribution to the D-term (2.32),

$$
0=f \mathbb{1}-g\left\langle\bar{\phi}^{-}\right\rangle\left\langle\phi^{-}\right\rangle .
$$

This is achieved by the expectation value

$$
\left\langle\phi^{-}\right\rangle=\sqrt{\frac{f}{g}} \sigma_{2} \otimes \mathbb{1}^{\prime} \equiv \sqrt{\frac{f}{g}} \hat{\sigma}_{2} .
$$

To find the remaining unbroken subgroup one has to determine the U(16) generators which commute with this expectation value,

$$
\left[\left\langle\hat{\phi}^{-}\right\rangle, \hat{V}\right]=\sqrt{2}\left(\begin{array}{cc}
0 & 0 \\
\left\langle\phi^{-}\right\rangle V+V^{T}\left\langle\phi^{-}\right\rangle & 0
\end{array}\right)=0 .
$$

One easily verifies that $\left\langle\hat{\phi}^{-}\right\rangle$commutes with $\hat{V}$ for $V=U$, i.e., $\left\langle\phi^{-}\right\rangle U+U^{T}\left\langle\phi^{-}\right\rangle=0$, where

$$
U: \quad i \sigma_{0} \otimes A^{\prime}, \quad \sigma_{1} \otimes S^{\prime}, \quad \sigma_{2} \otimes S^{\prime}, \quad \sigma_{3} \otimes S^{\prime} .
$$

These matrices generate the group $\operatorname{USp}(16)$ [30]; the related matrices $\hat{\sigma}_{2} U$ are asymmetric. The coset $\mathrm{U}(16) / \mathrm{USp}(16)$ is generated by $V=X$, where

$$
X: \quad \sigma_{0} \otimes S^{\prime}, \quad i \sigma_{1} \otimes A^{\prime}, \quad i \sigma_{2} \otimes A^{\prime}, \quad i \sigma_{3} \otimes A^{\prime} .
$$

The matrices $\hat{\sigma}_{2} X$ are antisymmetric, and one has

$$
\left\langle\phi^{-}\right\rangle X=X^{T}\left\langle\phi^{-}\right\rangle .
$$

The tachyonic instability will lead to a condensate of $\phi^{-}$, with a backreaction on the flux $\phi$, and in general also on $\phi^{+}$. Often times, it is argued that after tachyon condensation the magnetic flux vanishes, and that also supersymmetry is restored. Hence, one might think that the breaking from $\mathrm{SO}(32)$ to $\mathrm{USp}(16)$ can be realized by means of the vacuum expectation value (vev)

$$
\langle\hat{\phi}\rangle=\left(\begin{array}{cc}
\phi \mathbb{1} & \sqrt{2} \varphi_{+} \hat{\sigma}_{2} \\
\sqrt{2} \hat{\sigma}_{2} \varphi_{-} & -\phi \mathbb{1}
\end{array}\right),
$$

with $\bar{\partial} \phi=0$. For constant vev's $\phi, \varphi_{+}$and $\varphi_{-}$, this corresponds to the choice of a particular Wilson line.

Supersymmetric vacua are characterized by vanishing F- and D-terms. Assuming that only one chiral superfield, $\hat{\phi}^{3}=\hat{\phi}$, acquires a vev, all F-terms vanish automatically. The requirement of vanishing D-terms corresponds to the first-order differential equations

$$
\begin{aligned}
& \partial \bar{\phi}+\bar{\partial} \phi-\sqrt{2} g\left(\bar{\varphi}_{-} \varphi_{-}-\varphi_{+} \bar{\varphi}_{+}\right)=0, \\
& (\partial-\sqrt{2} g \phi) \bar{\varphi}_{+}+(\bar{\partial}+\sqrt{2} g \bar{\phi}) \varphi_{-}=0 .
\end{aligned}
$$


For constant vev's, $\phi=v_{0} / \sqrt{2}$ and $\varphi_{ \pm}=v_{ \pm} / \sqrt{2}$, eq. (3.10) becomes a constraint on the vev's,

$$
v_{0} v_{+}^{*}=v_{-} v_{0}^{*},
$$

which automatically solves eq. (3.9), $\left|v_{+}\right|^{2}=\left|v_{-}\right|^{2}$.

It is straightforward to compute the masses of fermions, vectors and scalars in a background of constant vev's. For the quadratic part of the superpotential one finds, with $\phi^{i}=\phi_{U}^{i}+\phi_{X}^{i}(i, j=1,2)$,

$$
\begin{aligned}
& W_{m} \supset-\frac{1}{4} \varepsilon_{i j} \int d^{2} z \operatorname{Tr} {\left[\hat{\phi}^{i}\left(\partial \hat{\phi}^{j}+\frac{g}{\sqrt{2}}\left[\langle\hat{\phi}\rangle, \hat{\phi}^{j}\right]\right)\right] } \\
&=-\frac{1}{2} \varepsilon_{i j} \int d^{2} z \operatorname{tr}\left[\phi_{U}^{i} \partial \phi_{U}^{j}+\phi_{X}^{i}\left(\partial \phi_{X}^{j}+g\left(v_{+} \phi^{j-}-v_{-} \phi^{k+}\right)\right)\right. \\
&\left.+2 \phi^{i+}\left(\partial-g v_{0}\right) \phi^{j-}+g\left(v_{-} \phi^{i+}-v_{+} \phi^{i-}\right) \phi_{X}^{j}\right] .
\end{aligned}
$$

The Kaluza-Klein mode functions are given by

$$
\lambda_{l, m}(z)=e^{z M_{l, m}-\bar{z} \bar{M}_{l, m}}, \quad M_{l, m}=2 \pi(m+i l) \equiv M_{\eta} .
$$

Introducing $\eta=(l, m)$, with $l>0, m \in \mathbb{Z}$ or $l=0, m>0$, and $\bar{\eta}=(-l,-m)$, one has $\lambda_{\bar{\eta}}=\bar{\lambda}_{\eta}$ and

$$
\partial \lambda_{\eta}=M_{\eta} \lambda_{\eta}, \quad \bar{\partial} \lambda_{\eta}=-\bar{M}_{\eta} \lambda_{\eta} .
$$

The mode expansion of a chiral superfield $\phi$ takes the form

$$
\phi=\phi_{0}+\sum_{\eta}\left(\phi_{\eta} \lambda_{\eta}+\phi_{\bar{\eta}} \lambda_{\bar{\eta}}\right) .
$$

Inserting this expansion into eq. (3.12) one finds

$$
W_{m}=-\varepsilon_{i j} \sum_{\eta} \operatorname{tr}\left[\phi_{U \bar{\eta}}^{i} M_{\eta} \phi_{U \eta}^{j}+\left(\phi_{X \bar{\eta}}^{i}, \phi_{\bar{\eta}}^{i-}, \phi_{\bar{\eta}}^{i+}\right) \mathcal{M}_{f}\left(\phi_{X \eta}^{j}, \phi_{\eta}^{j+}, \phi_{\eta}^{j-}\right)^{T}\right]
$$

where the fermion mass matrix is given by

$$
\mathcal{M}_{f}=\left(\begin{array}{ccc}
M_{\eta} & -g v_{-} & g v_{+} \\
-g v_{+} & M_{\eta}+g v_{0} & 0 \\
g v_{-} & 0 & M_{\eta}-g v_{0}
\end{array}\right)
$$

Its determinant factorizes as

$$
\operatorname{det} \mathcal{M}_{f}=M_{\eta}\left(M_{\eta}^{2}-g^{2}\left(v_{0}^{2}+2 v_{+} v_{-}\right)\right) .
$$

The matrix $\mathcal{M}_{f}$ describes the mixing of six antisymmetric tensors $\phi_{\eta}^{i \pm}$ and $\phi_{X \eta}^{i}, i=1,2$. One eigenvalue is $M_{\eta}$, the other two are $M_{\eta} \pm g \sqrt{v_{0}^{2}+2 v_{+} v_{-}}$. For $v_{ \pm}=0$ this corresponds to the standard spectrum of an Abelian Wilson line: for $M_{\eta}=0$, there is a pair of massless chiral multiplets; otherwise, always four chiral multiplets form degenerate Dirac mass terms. For $v_{ \pm} \neq 0$, there still is one pair of zero modes for $M_{\eta}=0$. This is surprising, since naively one would expect that the vev's of the antisymmetric tensors $\phi^{ \pm}$break $\mathrm{SO}(32)$ 
to $\mathrm{USp}(16)$, as discussed above, and all multiplets become massive. However, the massive spectrum corresponds to an unbroken group $\mathrm{U}(16)$. We conclude that for any values $v_{0}, v_{+}$ and $v_{-}$that satisfy eq. (3.11), the vacuum expectation value (3.8) represents an Abelian Wilson line, just with different embeddings into $\mathrm{SO}(32)$. The scalar mass matrix is given by

$$
\begin{aligned}
\mathcal{M}_{S}^{2} & =\mathcal{M}_{f}^{\dagger} \mathcal{M}_{f} \\
& =\left(\begin{array}{ccc}
\left|M_{\eta}\right|^{2}+2 g^{2}\left|v_{+}\right|^{2} & -g\left(v_{-} \bar{M}_{\eta}+v_{+}^{*} M_{\eta+}\right) & g\left(v_{+} \bar{M}_{\eta}+v_{-}^{*} M_{\eta-}\right) \\
-g\left(v_{-}^{*} M_{\eta}+v_{+} \bar{M}_{\eta+}\right) & \left|M_{\eta+}\right|^{2}+g^{2}\left|v_{+}\right|^{2} & -g^{2} v_{-}^{*} v_{+} \\
g\left(v_{+}^{*} M_{\eta}+v_{-} \bar{M}_{\eta-}\right) & -g^{2} v_{+}^{*} v_{-} & \left|M_{\eta-}\right|^{2}+g^{2}\left|v_{+}\right|^{2}
\end{array}\right),
\end{aligned}
$$

where the relations $v_{0} v_{+}^{*}=v_{-} v_{0}^{*}$ and $\left|v_{+}\right|^{2}=\left|v_{-}\right|^{2}$ have been used, and $M_{\eta \pm} \equiv M_{\eta} \pm g v_{0}$.

In order to verify that supersymmetry is unbroken one also has to evaluate the vector boson masses. Analogous to the superpotential terms, the starting point is now

$$
\begin{aligned}
& \partial \hat{V}+\frac{g}{\sqrt{2}}[\langle\hat{\phi}\rangle, \hat{V}]= \\
& \quad\left(\begin{array}{cc}
\partial U+\partial X+g\left(v_{+} X^{-}-v_{-} X^{+}\right) & \sqrt{2}\left(\left(\partial+g v_{0}\right) X^{+}-g v_{+} X\right) \hat{\sigma}_{2} \\
\sqrt{2} \hat{\sigma}_{2}\left(\left(\partial-g v_{0}\right) X^{-}+g v_{-} X\right) & -\left(\partial U+\partial X+g\left(v_{+} X^{-}-v_{-} X^{+}\right)\right)^{T}
\end{array}\right) .
\end{aligned}
$$

Here we have introduced the fields

$$
V^{-}=\hat{\sigma}_{2} X^{-}, \quad V^{+}=X^{+} \hat{\sigma}_{2}, \quad X^{-}=X^{+\dagger},
$$

and we have used the relation (3.7), $\hat{\sigma}_{2} X^{T}=X \hat{\sigma}_{2}$. The part of the Kaehler potential containing the vector-boson masses is given by

$$
K_{m}=\frac{1}{2} \int d^{2} z \operatorname{Tr}\left[\left(\partial \hat{V}+\frac{g}{\sqrt{2}}[\langle\hat{\phi}\rangle, \hat{V}]\right)^{\dagger}\left(\partial \hat{V}+\frac{g}{\sqrt{2}}[\langle\hat{\phi}\rangle, \hat{V}]\right)\right] .
$$

In the KK mode expansion (see eq. (3.13)) one has to make sure that the relations $X^{\dagger}=X$ and $X^{+\dagger}=X^{-}$are satisfied, which yields

$$
\begin{aligned}
X & =X_{0}+\sum_{\eta}\left(X_{\eta} \lambda_{\eta}+X_{\bar{\eta}} \lambda_{\bar{\eta}}\right), & X_{\bar{\eta}} & =X_{\eta}^{\dagger}, \\
X^{ \pm} & =X_{0}^{ \pm}+\sum_{\eta}\left(X_{\eta}^{ \pm} \lambda_{\eta}+X_{\bar{\eta}}^{ \pm} \lambda_{\bar{\eta}}\right), & X_{\eta}^{ \pm \dagger} & =X_{\bar{\eta}}^{\mp} .
\end{aligned}
$$

Inserting this expansion into eq. (3.22) one finds

$$
K_{m}=2 \sum_{\eta} \operatorname{tr}\left[\left|M_{\eta}\right|^{2} U_{\bar{\eta}} U_{\eta}+\left(X_{\bar{\eta}}, X_{\bar{\eta}}^{-}, X_{\bar{\eta}}^{+}\right) \mathcal{M}_{V}^{2}\left(X_{\eta}, X_{\eta}^{+}, X_{\eta}^{-}\right)\right],
$$

where the vector-boson mass matrix indeed satisfies

$$
\mathcal{M}_{V}^{2}=\mathcal{M}_{f}^{\dagger} \mathcal{M}_{f}=\mathcal{M}_{S}^{2}
$$

A pair of massive $4 \mathrm{~d} \mathcal{N}=1$ vector multiplets is mass degenerate with four $4 \mathrm{~d} \mathcal{N}=1$ chiral multiplets, corresponding altogether to eight Weyl fermions, six complex scalars and a complex vector. These degrees of freedom form a massive $8 \mathrm{~d} \mathcal{N}=1$ multiplet. The massless multiplet contains half the number of degrees of freedom. We conclude that Wilson lines can break $\mathrm{SO}(32)$ only to $\mathrm{U}(16)$, but not to $\mathrm{USp}(16)$. 


\section{Dilute vs. singular flux}

In the magnetic flux background discussed in section 2, only the fields $\phi_{0, j}^{-}$are tachyonic where $j=0, \ldots, 2 k-1$ labels the degeneracy for $k$ flux quanta. This suggests to search for solutions of the equations of motion where only $\phi_{0, j}^{-}$acquire a non-zero vev. Searching for solutions with unbroken supersymmetry, we again consider the first-order differential equations corresponding to vanishing D-terms, which now read $\left(\left\langle\phi_{0, j}^{-}\right\rangle=\hat{\sigma}_{2} \varphi_{-, j}, \varphi_{-, j} \equiv \varphi_{j}\right)$

$$
\partial \bar{\phi}+\bar{\partial} \phi-\sqrt{2} g \sum_{j=0}^{2 k-1} \bar{\varphi}_{j} \varphi_{j}=0, \quad(\bar{\partial}+\sqrt{2} g \bar{\phi}) \varphi_{j}=0 .
$$

First, we consider these equations on the covering space $\mathbb{C}$. The equation for $\varphi_{j}$ can be solved with the ansatz ${ }^{8}$

$$
\phi=\frac{1}{\sqrt{2}} \partial \chi, \quad \varphi_{0}=\varphi=\beta z^{b} e^{-g \chi}, \quad \varphi_{j \geq 1}=0, \quad \chi=\bar{\chi},
$$

which leads to a second-order differential equation for $\chi$,

$$
\partial \bar{\partial} \chi=g \beta^{2}(\bar{z} z)^{b} e^{-2 g \chi} .
$$

After introducing polar coordinates, $\partial \bar{\partial}=r^{-1} \partial_{r}\left(r \partial_{r}\right)+r^{-2} \partial_{\theta}^{2}$, changing $r$ to $t=\ln r$, and defining $h=\chi-(b+1) t / g$, this becomes

$$
\partial_{t}^{2} h(t)=g \beta^{2} e^{-2 g h(t)} .
$$

A straightforward calculation yields the result

$$
g h=\ln \left\{\frac{\beta}{2^{b+1} C}\left[\left(\frac{|z|}{\left|z_{0}\right|}\right)^{g C}+\left(\frac{\left|z_{0}\right|}{|z|}\right)^{g C}\right]\right\},
$$

where $C>0$ and $\left|z_{0}\right|$ are integration constants. This yields for $g \chi=g h+(b+1) t$,

$$
g \chi=\ln \left\{\frac{\beta\left|z_{0}\right|^{b+1}}{C}\left[\left(\frac{|z|}{\left|z_{0}\right|}\right)^{g C+b+1}+\left(\frac{\left|z_{0}\right|}{|z|}\right)^{g C-b-1}\right]\right\} .
$$

Demanding that the solution is regular at $z=0$ fixes the integration constant $C$ as $C=$ $(b+1) / g$. One then obtains for the fields $\phi$ and $\varphi$

$$
\begin{aligned}
& \phi=\frac{b+1}{\sqrt{2} g z} \frac{|z|^{2(b+1)}}{\left|z_{0}\right|^{2(b+1)}+|z|^{2(b+1)}}, \\
& \varphi=\frac{b+1}{g} z^{b} \frac{\left|z_{0}\right|^{b+1}}{\left|z_{0}\right|^{2(b+1)}+|z|^{2(b+1)}} .
\end{aligned}
$$

One might think that the flux could somehow disappear in the process of tachyon condensation. This would be the case if, by varying continuously a parameter, the solution

\footnotetext{
${ }^{8}$ Such an ansatz has previously been considered for zero-mode wave functions on a magnetized orbifold [31, 32].
} 
interpolates between the magnetic field and the usual vacuum, with no magnetic flux and no tachyon condensation. This is however not the case. For general integration constant $C$ the classical solution is singular, so $C$ cannot vary continuously. The trivial vacuum solution, with no magnetic flux, is of course also a solution of the D-term equations (4.1). But it is an isolated solution, not connected by any parameter deformation to the classical solution (4.7).

The constant $z_{0}$ defines a length scale that separates the complex plane into an inner core, $|z|<\left|z_{0}\right|$ and an outer region, $|z|>\left|z_{0}\right|$. For $b=0$, one obtains in the inner core $|z| \ll\left|z_{0}\right|$ a constant flux density and constant expectation value $\varphi$ (cf. eqs. (2.15), (3.3)),

$$
\phi=\frac{f \bar{z}}{\sqrt{2}}\left(1+\mathcal{O}\left(\frac{|z|^{2}}{\left|z_{0}\right|^{2}}\right)\right), \quad \varphi=\sqrt{\frac{f}{g}}\left(1+\mathcal{O}\left(\frac{|z|^{2}}{\left|z_{0}\right|^{2}}\right)\right),
$$

where $f=1 /\left(g\left|z_{0}\right|^{2}\right)$. Note that for this perturbative solution in powers of $|z|^{2} /\left|z_{0}\right|^{2}$ the D-term vanishes.

The radius $\left|z_{0}\right|$ plays the role of a regulator and the limit $\left|z_{0}\right| \rightarrow 0$ is of particular interest. In this limit $\phi$ and $\varphi$ are singular and from eq. (4.7) one obtains

$$
\phi \rightarrow \frac{b+1}{\sqrt{2} g z}, \quad \varphi^{2} \rightarrow \frac{4(b+1) \pi}{g^{2}} \delta^{(2)}(z, \bar{z}) .
$$

$\phi=\left(A_{9}+i A_{8}\right) / \sqrt{2}$ describes the vortex vector potential,

$$
A_{m}=-\frac{2(b+1)}{g} \epsilon_{m n} \frac{x_{n}}{x_{8}^{2}+x_{9}^{2}}
$$

The flux vanishes everywhere except at the origin, and the localized flux density is given by a line integral over a 1 -cycle $\mathcal{C}_{O}$ around the origin, ${ }^{9}$

$$
g \oint_{\mathcal{C}_{O}} A=4 \pi(b+1) .
$$

On the plane the flux is not quantized and therefore $b$ can take arbitrary values. On the torus flux quantization requires $b+1=k / 2, k \in \mathbb{Z}$, which will be discussed in the next section. Note that a singular wave function ${ }^{10} \varphi \propto \delta^{1 / 2}$ has previously been considered in connection with the infinite flux limit on a magnetized torus [33].

Notice that for $b \neq 0$ the classical solution has no rotational symmetry around the core $z=0$. Also, while in the zero-size limit $z_{0} \rightarrow 0$ the solution has the same localized flux interpretation for any $b$, in the opposite limit $z_{0} \gg z$ it does not correspond anymore to a constant magnetic field unless $b=0$. Based on the string theory intuition in section 6 and on the analogy with instanton solutions, we believe that for a general magnetic flux, the interpolation between a constant magnetic field and the localized flux should start from a multicenter solution, with a different ansatz. We were unable to find explicitly the solution but hope to come back to this point in the future.

\footnotetext{
${ }^{9}$ Since the charge of the antisymmetric tensor field is $q=2$, the factor $4 \pi$ occurs instead of $2 \pi$.

${ }^{10}$ We use the notation $\delta^{(2)}(z, \bar{z}) \equiv \delta\left(x_{8}, x_{9}\right)$.
} 


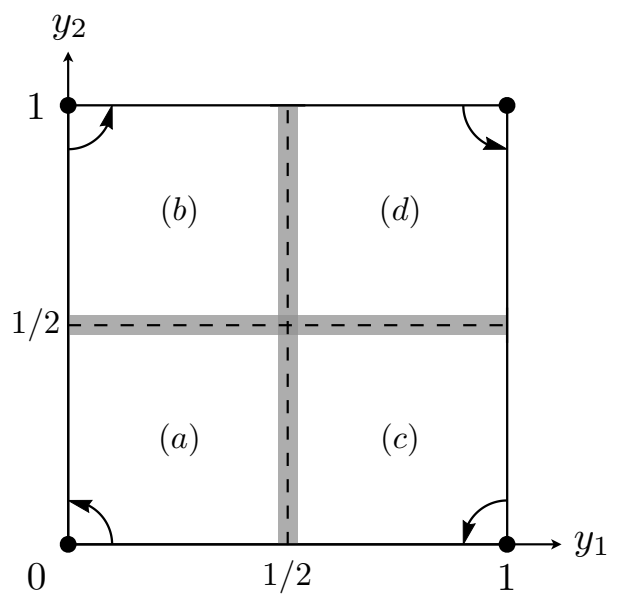

Figure 1. Vortex vector potential with singularity at $\left(y_{1}, y_{2}\right)=(0,0)$ on a square torus covered by four patches.

It is often assumed that tachyon condensation corresponds to the annihilation of two branes such that the total magnetic flux vanishes. Our result is different. We find that the total flux of the unstable state is still present after tachyon condensation, but it is squeezed into a singularity. As we shall see below, this affects the boundary conditions of fields in this vacuum and therefore the massive spectrum.

Finally, since this classical solution was obtained by ignoring the gravitational sector, one might worry about the gravitational backreaction that could change it significantly. However, the Yang-Mills classical solution is supersymmetric and its energy is zero. Therefore the gravitational fields will not be excited and the spacetime geometry remains flat.

\section{$5 \quad$ Massive spectrum for singular flux}

In the previous section we have constructed a solution of the equations of motion for $\phi$ and $\phi^{-}$in the complex plane. Let us now consider the singular limit of this solution as background fields on the torus. The gauge background $\phi$ corresponds to the vortex vector potential depicted in figure 1 . The field $\phi^{-}$is localized at the origin.

The vector potential can be expressed in terms of the singular function ${ }^{11}$

$$
\Lambda\left(y_{1}, y_{2}\right)=i \frac{k}{2 g} \ln \left(\frac{y_{1}+i y_{2}}{y_{1}-i y_{2}}\right)=\bar{\Lambda}\left(y_{1}, y_{2}\right)
$$

where $k=2(b+1)$ is determined by the flux density (see eq. (4.10)). In the four patches one has (see eq. (4.10))

$$
\begin{aligned}
& \text { (a) } \quad A_{m}^{a}\left(y_{1}, y_{2}\right)=-\partial_{m} \Lambda_{a}\left(y_{1}, y_{2}\right), \quad \Lambda_{a}\left(y_{1}, y_{2}\right)=\Lambda\left(y_{1}, y_{2}\right) \text {, } \\
& \text { (b) } A_{m}^{b}\left(y_{1}, y_{2}\right)=-\partial_{m} \Lambda_{b}\left(y_{1}, y_{2}\right), \quad \Lambda_{b}\left(y_{1}, y_{2}\right)=\Lambda\left(y_{1}, y_{2}-1\right) \text {, } \\
& \text { (c) } \quad A_{m}^{c}\left(y_{1}, y_{2}\right)=-\partial_{m} \Lambda_{c}\left(y_{1}, y_{2}\right), \quad \Lambda_{c}\left(y_{1}, y_{2}\right)=\Lambda\left(y_{1}-1, y_{2}\right) \text {, } \\
& \text { (d) } \quad A_{m}^{d}\left(y_{1}, y_{2}\right)=-\partial_{m} \Lambda_{d}\left(y_{1}, y_{2}\right), \quad \Lambda_{d}\left(y_{1}, y_{2}\right)=\Lambda\left(y_{1}-1, y_{2}-1\right) \text {. }
\end{aligned}
$$

\footnotetext{
${ }^{11}$ For convenience, we now rescale $A_{8,9} \rightarrow A_{1,2} /(2 \pi R), x_{8,9} \rightarrow 2 \pi R y_{1,2}$.
} 
The transition functions are easily obtained from eqs. (5.1), (5.2) and in appendix A (A.2). For example, for the patches $(a)$ and $(b)$ one has

$$
\left(\partial_{m} \Lambda_{b a}\right)\left(y_{1}, 1 / 2\right)=g\left(A_{m}^{a}\left(y_{1}, 1 / 2\right)-A_{m}^{b}\left(y_{1}, 1 / 2\right)\right)=-g\left(\partial_{m} \Lambda_{a}-\partial_{m} \Lambda_{b}\right)\left(y_{1}, 1 / 2\right),
$$

which yields, up to a constant,

$$
\begin{aligned}
\Lambda_{b a}\left(y_{1}, 1 / 2\right) & =-g\left(\Lambda_{a}\left(y_{1}, 1 / 2\right)-\Lambda_{b}\left(y_{1}, 1 / 2\right)\right) \\
& =i k \ln \left(\frac{y_{1}-i / 2}{y_{1}+i / 2}\right)=2 k \arctan \left(1 /\left(2 y_{1}\right)\right) .
\end{aligned}
$$

The charged fields in the patches $(a)$ and $(b)$ are related by the transition function,

$$
\phi_{b}^{-}\left(y_{1}, 1 / 2\right)=S_{b a}^{-1}\left(y_{1}, 1 / 2\right) \phi_{a}^{-}\left(y_{1}, 1 / 2\right)=e^{-i \Lambda_{b a}\left(y_{1}, 1 / 2\right)} \phi_{a}^{-}\left(y_{1}, 1 / 2\right) .
$$

For the singular solution the charged field is localized, ${ }^{12} \phi_{a}^{-}\left(y_{1}, y_{2}\right) \propto \delta^{1 / 2}\left(y_{1}, y_{2}\right)$. Analogously, in patch $(b)$ one has $\phi_{b}^{-}\left(y_{1}, y_{2}\right) \propto \delta^{1 / 2}\left(y_{1}, y_{2}-1\right)$. Hence, in the singular limit the field $\phi^{-}$vanishes in the bulk and the condition (5.5) is trivially satisfied. The same holds for all boundaries between different patches, which means that the singular background fields represent a solution of the equations of motion on the torus. The consistency condition at the overlap of the four patches,

$$
S_{a c}(1 / 2,1 / 2) S_{c d}(1 / 2,1 / 2) S_{d b}(1 / 2,1 / 2) S_{b a}(1 / 2,1 / 2)=e^{-i 2 \pi k}=1,
$$

implies $k \in \mathbb{Z}$ and therefore standard flux quantization also for the localized flux.

In order to obtain the massive spectrum of the $8 \mathrm{~d}$ theory one has to shift in the 10d Lagrangian (2.14) the fields $\phi$ and $\phi^{-}$around the background fields $\langle\phi\rangle$ and $\left\langle\phi^{-}\right\rangle$, respectively, and keep the quadratic part of the Lagrangian, which reads

$$
\begin{aligned}
\mathcal{L}_{m} \supset \int d^{4} \theta \operatorname{tr}[ & \left(\bar{\partial} V-\sqrt{2} g\left\langle\bar{\phi}^{-}\right\rangle V^{-}\right)\left(\partial V-\sqrt{2} g V^{+}\left\langle\phi^{-}\right\rangle\right) \\
+ & \left(\bar{\partial} V^{+}-\sqrt{2} g\langle\bar{\phi}\rangle V^{+}+\frac{g}{\sqrt{2}}\left(V\left\langle\bar{\phi}^{-}\right\rangle+\left\langle\bar{\phi}^{-}\right\rangle V^{T}\right)\right) \\
& \times\left(\partial V^{-}-\sqrt{2} g\langle\phi\rangle V^{-}+\frac{g}{\sqrt{2}}\left(\left\langle\phi^{-}\right\rangle V+V^{T}\left\langle\phi^{-}\right\rangle\right)\right) \\
+ & \left.\left(\bar{\partial} V^{-}+\sqrt{2} g\langle\bar{\phi}\rangle V^{-}\right)\left(\partial V^{+}+\sqrt{2} g\langle\phi\rangle V^{+}\right)\right] .
\end{aligned}
$$

With $V=U+X$, where $U \in \mathrm{USp}(16)$ and $X \in \mathrm{U}(16) / \mathrm{USp}(16)$, one has

$$
V\left\langle\bar{\phi}^{-}\right\rangle+\left\langle\bar{\phi}^{-}\right\rangle V^{T}=2 X\left\langle\bar{\phi}^{-}\right\rangle, \quad \operatorname{tr}\left[U V^{+}\left\langle\phi^{-}\right\rangle\right]=0 .
$$

Therefore $U$ does not mix with charged fields, and defining $\left\langle\bar{\phi}^{-}\right\rangle=\hat{\sigma}_{2} \varphi, V^{-}=X^{-} \hat{\sigma}_{2}$ and $V^{+}=\hat{\sigma}_{2} X^{+}$, one obtains

$$
\begin{aligned}
\mathcal{L}_{m} \supset \int d^{4} \theta \operatorname{tr}[ & \bar{\partial} U \partial U+\left(\bar{\partial} X-\sqrt{2} g \varphi X^{-}\right)\left(\partial X-\sqrt{2} g \bar{\varphi} X^{+}\right) \\
& +\left((\bar{\partial}-\sqrt{2}\langle\bar{\phi}\rangle) X^{+}+\sqrt{2} g \varphi X\right)\left((\partial-\sqrt{2} g\langle\phi\rangle) X^{-}+\sqrt{2} g \bar{\varphi} X\right) \\
& \left.+(\bar{\partial}+\sqrt{2} g\langle\bar{\phi}\rangle) X^{-}(\partial+\sqrt{2} g\langle\phi\rangle) X^{+}\right] .
\end{aligned}
$$

\footnotetext{
${ }^{12}$ We use the notation $\delta^{(2)}(z, \bar{z}) \equiv \delta\left(y_{1}, y_{2}\right)$.
} 
In the background gauge field $\langle\phi\rangle$, the charged fields $X^{ \pm}$are also defined in the four patches. At the overlaps they are related by gauge transformations, for instance,

$$
X_{b}^{+}\left(y_{1}, 1 / 2\right)=S_{b a}\left(y_{1}, 1 / 2\right) X_{a}^{+}\left(y_{1}, 1 / 2\right)=e^{i \Lambda_{b a}\left(y_{1}, 1 / 2\right)} X_{a}^{+}\left(y_{1}, 1 / 2\right) .
$$

The singular vector field defined in eq. (5.2) can be removed by a singular redefinition of the charged fields

$$
X_{a}^{+}\left(y_{1}, y_{2}\right)=e^{i g \Lambda_{a}\left(y_{1}, y_{2}\right)} \hat{X}_{a}^{+}\left(y_{1}, y_{2}\right), \quad X_{b}^{+}\left(y_{1}, y_{2}\right)=e^{i g \Lambda_{b}\left(y_{1}, y_{2}\right)} \hat{X}_{b}^{+}\left(y_{1}, y_{2}\right), \ldots
$$

The new fields have trivial transition functions, ${ }^{13}$ e.g.,

$$
\begin{aligned}
\hat{X}_{b}^{+}\left(y_{1}, 1 / 2\right) & =e^{-i g \Lambda_{b}\left(y_{1}, 1 / 2\right)} X_{b}^{+}\left(y_{1}, 1 / 2\right)=e^{-i g \Lambda_{b}\left(y_{1}, 1 / 2\right)+i \Lambda_{b a}\left(y_{1}, 1 / 2\right)} X_{a}^{+}\left(y_{1}, 1 / 2\right) \\
& =e^{-i g \Lambda_{a}\left(y_{1}, 1 / 2\right)} X_{a}^{+}\left(y_{1}, 1 / 2\right)=\hat{X}_{a}^{+}\left(y_{1}, 1 / 2\right) .
\end{aligned}
$$

In terms of the fields $\hat{X}^{ \pm}$the Lagrangian (5.9) becomes

$$
\begin{aligned}
\mathcal{L}_{m} \supset \int d^{4} \theta \operatorname{tr}\left[\bar{\partial} U \partial U+\left(\bar{\partial} X-\sqrt{2} g \varphi^{\prime} \hat{X}^{-}\right)\left(\partial X-\sqrt{2} g \bar{\varphi}^{\prime} \hat{X}^{+}\right)\right. \\
\left.+\left(\bar{\partial} \hat{X}^{+}+\sqrt{2} g \varphi^{\prime} X\right)\left(\partial \hat{X}^{-}+\sqrt{2} g \bar{\varphi}^{\prime} X\right)+\bar{\partial} \hat{X}^{-} \partial \hat{X}^{+}\right],
\end{aligned}
$$

where $\varphi^{\prime}=\varphi \exp \left(i \Lambda_{O}\right)$ is the redefined charged background field that is singular at the origin $O$.

Except for the singular term at the origin, this Lagrangian describes free fields. Due to the singular term, the equations of motion require that the fields $X$ and $\hat{X}^{ \pm}$vanish at the origin. A basis of periodic $(P)$ and anti-periodic $(A)$ functions on the interval $[0,1]$ is given by $(m \in \mathbb{Z})$,

$$
\begin{array}{lll}
\text { (P) } & c(x)=\cos (2 \pi m x), & s(x)=\sin (2 \pi m x), \\
(A) & \hat{c}(x)=\cos \left(2 \pi\left(m+\frac{1}{2}\right) x\right), & \hat{s}(x)=\sin \left(2 \pi\left(m+\frac{1}{2}\right) x\right) .
\end{array}
$$

From combinations of these periodic and anti-periodic functions one can form various products that vanish at the origin of the torus. On the other hand, $U$ has a zero mode and its wavefunctions need not vanish at the origin. One natural way to achieve this is to assign $U$ an even parity under reflection of torus coordinates, whereas $X, \hat{X}^{ \pm}$are assigned odd parity. An example of mode functions for the four real fields $U, X, X_{1}=\left(\hat{X}^{+}+\hat{X}^{-}\right) / \sqrt{2}$ and $X_{2}=i\left(\hat{X}^{+}-\hat{X}^{-}\right) / \sqrt{2}$, exhausting all possibilities, is given by

$$
\begin{array}{rll}
U: & c\left(y_{1}\right) c\left(y_{2}\right), & s\left(y_{1}\right) s\left(y_{2}\right), \\
X_{1}: & \hat{c}\left(y_{1}\right) s\left(y_{2}\right), & \hat{s}\left(y_{1}\right) c\left(y_{2}\right), \\
X_{2}: & c\left(y_{1}\right) \hat{s}\left(y_{2}\right), & s\left(y_{1}\right) \hat{c}\left(y_{2}\right), \\
X: & \hat{c}\left(y_{1}\right) \hat{s}\left(y_{2}\right), & \hat{s}\left(y_{1}\right) \hat{c}\left(y_{2}\right) .
\end{array}
$$

\footnotetext{
${ }^{13}$ This is analogous to the transition from 'twisted' wave functions to 'untwisted' wave functions on a magnetized torus, as discussed in ref. [32].
} 
Note that $U$ is periodic, whereas $X_{1}, X_{2}$ and $X$ satisfy twisted boundary conditions in $y_{1}$-direction, $y_{2}$-direction and both directions, respectively. The mass spectra $M_{m_{1}, m_{2}}^{2} R^{2}$ in the four cases are given by

$$
\begin{array}{clll}
U: & m_{1}^{2}+m_{2}^{2}, & X: & \left(m_{1}+\frac{1}{2}\right)^{2}+\left(m_{2}+\frac{1}{2}\right)^{2}, \\
X_{1}: & \left(m_{1}+\frac{1}{2}\right)^{2}+m_{2}^{2}, & X_{2}: & m_{1}^{2}+\left(m_{2}+\frac{1}{2}\right)^{2} .
\end{array}
$$

The boundary conditions (5.15) only represent an example. To determine them uniquely, information beyond the Lagrangian (5.13) is required, involving interaction terms between the fields and possibly also couplings to string excitations.

\section{An alternative D9-D7 brane description}

In section 4 we noticed that the classical solution with tachyon condensation has a singular limit when the length of the core supporting the solution shrinks to zero size. The resulting singular solution has a delta-function like flux, which in string theory has the natural interpretation of a D7-brane. In the following we therefore recall the connection between a magnetized D9-brane and a system of elementary D9-D7 branes in type I string theory.

In type IIB, a D9-brane with a magnetic field in its worldvolume generates a D7-brane charge from the Wess-Zumino coupling

$$
\int C \wedge e^{F}=\int C_{10}+\int C_{8} \wedge F
$$

where $C_{p+1}$ denote the $\mathrm{RR}(p+1)$-form fields and $F$ is the magnetic 2 -form field. In type I strings $C_{8}$ is removed by the orientifold projection $\Omega$. This implies that the BPS D7-brane of type IIB string becomes the non-BPS (uncharged) D7-brane of type I, see [34] for more details of its construction. So let us start from the corresponding system of D9and non-BPS D7-branes in type I. The gauge group for the D9-branes and a number $d$ of D7-branes is $\mathrm{SO}(N)_{9} \times \mathrm{U}(d)_{7}$, with $N=32$ due to the RR tadpole condition in type I. Whereas $d$ could in principle be arbitrary since the non-BPS D7-branes have no RR charge, only stacks with $d \leq 16$ are related to the magnetized D9-brane picture, as will be explained below. The low-energy massless and tachyonic string spectrum, ignoring massive string excitations, comprises [34]

- supersymmetric gauge multiplets $\left(\frac{\mathbf{N}(\mathbf{N}-\mathbf{1})}{\mathbf{2}}, \mathbf{1}\right)+(\mathbf{1}, \mathbf{d} \overline{\mathbf{d}})$,

- chiral fermions $\left(\mathbf{1}, \frac{\mathbf{d}(\mathbf{d}-\mathbf{1})}{\mathbf{2}}\right)_{R}+\left(\mathbf{1}, \frac{\mathbf{d}(\mathbf{d}+\mathbf{1})}{\mathbf{2}}\right)_{L}+(\mathbf{N}, \mathbf{d})_{R}$,

- complex scalar tachyons $\left(\mathbf{1}, \frac{\mathbf{d}(\mathbf{d}-\mathbf{1})}{\mathbf{2}}\right)+(\mathbf{N}, \mathbf{d})$.

The spectrum can be found in a systematic way from the partition functions [34], with the light-cone $\mathrm{SO}(8)$ characters decomposed appropriately into products of $\mathrm{SO}(6)$ and $\mathrm{SO}(2)$ 
characters. Using notations and conventions of $[2,24]$, the corresponding amplitudes are given by

$$
\begin{gathered}
\mathcal{A}_{99}=\frac{N^{2}}{2} \int_{0}^{\infty} \frac{d \tau_{2}}{\tau_{2}^{5}} \frac{1}{\eta^{8}}\left(V_{6} O_{2}+O_{6} V_{2}-S_{6} S_{2}-C_{6} C_{2}\right) P_{0}^{(1)} P_{0}^{(2)} \\
\mathcal{A}_{77}=\int_{0}^{\infty} \frac{d \tau_{2}}{\tau_{2}^{5}} \frac{1}{\eta^{8}}\left(d \bar{d}\left(V_{6} O_{2}+O_{6} V_{2}-S_{6} S_{2}-C_{6} C_{2}\right)\right. \\
\left.\quad+\frac{d^{2}+\bar{d}^{2}}{2}\left(O_{6} O_{2}+V_{6} V_{2}-S_{6} C_{2}-C_{6} S_{2}\right)\right) W_{0}^{(1)} W_{0}^{(2)} \\
\mathcal{A}_{79}=\int_{0}^{\infty} \frac{d \tau_{2}}{\tau_{2}^{5}} \frac{1}{\theta_{4} \eta^{5}}\left(N d\left(O_{6} S_{2}+V_{6} C_{2}-C_{6} O_{2}-S_{6} V_{2}\right)\right. \\
\left.\quad+N \bar{d}\left(O_{6} C_{2}+V_{6} S_{2}-S_{6} O_{2}-C_{6} V_{2}\right)\right) \\
\mathcal{M}_{9}=-\frac{N}{2} \int_{0}^{\infty} \frac{d \tau_{2}}{\tau_{2}^{5}} \frac{1}{\hat{\eta}^{8}}\left(\hat{V}_{6} \hat{O}_{2}+\hat{O}_{6} \hat{V}_{2}-\hat{S}_{6} \hat{S}_{2}-\hat{C}_{6} \hat{C}_{2}\right) P_{0}^{(1)} P_{0}^{(2)} \\
\mathcal{M}_{7}=-\frac{1}{2} \int_{0}^{\infty} \frac{d \tau_{2}}{\tau_{2}^{5}} \frac{2}{\hat{\theta}_{2} \hat{\eta}^{5}}\left((d+\bar{d})\left(\hat{O}_{6} \hat{O}_{2}+\hat{V}_{6} \hat{V}_{2}\right)\right. \\
\left.\quad+(d-\bar{d})\left(\hat{S}_{6} \hat{C}_{2}-\hat{C}_{6} \hat{S}_{2}\right)\right) W_{0}^{(1)} W_{0}^{(2)}
\end{gathered}
$$

where $P_{a}^{(i)}$ and $W_{a}^{(i)}$, with $i=1,2$ and $a=0,1 / 2$, are the momentum and winding sums, respectively.

The connection between the D9-D7 system and the magnetized D9-brane in type I can be understood in the following way. Start with type I with an equal number of D9- and D7branes, with gauge group $\mathrm{SO}(32)_{9} \times \mathrm{U}(16)_{7}$. By one T-duality, one gets the type I' string with 16 BPS D8-branes, with wrapping numbers $(1,0)$, and 16 non-BPS D8-branes with wrapping numbers $(0,1)$. The condensation of the bi-fundamental tachyon $(\mathbf{N}, \mathbf{d})$ breaks the gauge group $\mathrm{SO}(32)_{9} \times \mathrm{U}(16)_{7}$ to the diagonal $\mathrm{U}(16)$. This can be understood as a process of recombining the two D8-branes, with the recombined brane defined by a cycle with wrapping numbers $(1,1)$. At this step, this setup has the interpretation of intersecting D8-branes in type IIA in eight dimensions, which T-dualized back to type I correspond to a magnetized stack of 16 D9-branes, of minimum magnetic flux $k=1$ (see left part of figure 2).

Similarly, the more general case of magnetized D9-branes in type I with an arbitrary flux integer $k$ has the following description. One starts in type I with D9-branes and $k$ stacks of $M$ coincident non-BPS D7-branes, $k \leq 16$, at different locations on the 2 -torus. The initial gauge group is therefore $\mathrm{SO}(32) \times \mathrm{U}(M)^{k}$. In the large torus volume limit, there are tachyons in the bi-fundamental representation of $\mathrm{SO}(32)$ with all the $\mathrm{D} 7$ stacks, but the scalars stretched between different stacks of D7-branes are massive. After one T-duality, one obtains the type I' string with 16 BPS D8-branes, with wrapping numbers $(1,0)$ and $k$ parallel stacks of $M$ non-BPS $\mathrm{D} 8_{i}^{\prime}$-branes with wrapping numbers $(0,1)$. The condensation of the bi-fundamental tachyons stretched between the $\mathrm{D} 8$ - and $\mathrm{D} 8_{i}^{\prime}$-branes break the gauge group $\mathrm{SO}(32)_{9} \times \mathrm{U}(M)^{k}$ to $\mathrm{SO}(32-2 M) \times \mathrm{U}(M)$. In this case the recombined branes of gauge group $\mathrm{U}(M)$ have the wrapping numbers $(1, k)$. After T-dualizing back to type 
I, one obtains $16-M$ standard (unmagnetized) D9-branes and a magnetized stack of $M$ D9-branes, with magnetic flux $k$. The case of interest for this paper is when all D9-branes are magnetized, i.e. $M=16$.

The situation is conceptually similar to the D9-D5 or the D5-D1 brane systems first worked out in $[35,36]$. The D9-D5 system for example has three different but related descriptions. In one of them, the $k$ D5-branes are seen as configurations of instanton number $k$ on the D9-brane gauge theory, in the limit where the instanton size goes to zero. In $\mathrm{SO}(32)$ type I strings, the D5 gauge group is generically $\mathrm{USp}(2 k)$, and the moduli space coincides precisely with the one of the gauge theory instanton configurations in the $\mathrm{SO}(32)$ D9-brane gauge group. The instanton size is a modulus in the supersymmetric gauge theory, which can therefore be varied with no cost. For a finite instanton size, the D5-brane is not dynamical anymore. In the maximal instanton-size limit, the physics is captured by a smooth constant magnetic field configuration in the four dimensions transverse to the D5brane, of self-dual instanton type $B_{1}=B_{2}$, where $B_{1,2}=k_{1,2} / v_{i}$ are the constant magnetic fields in the two tori of area $v_{i}$.

In type I string theory, a D9-brane with magnetic fields in its worldvolume generates a D5-brane charge from the Wess-Zumino coupling

$$
\int C \wedge e^{F}=\int C_{10}+\int C_{6} \wedge F_{1} \wedge F_{2},
$$

where $F_{1,2}=B_{1,2} Q, Q$ being an Abelian generator of $\mathrm{SO}(32)$. The induced D5 charge in this case is $k=k_{1} k_{2}$. It is possible to write down explicitly the classical solution of the D9-D5 system and check the interpolation between the zero-size instanton limit, with D5-branes corresponding to a singular source in the limit, and the maximum size limit, where the solution describes self-dual constant magnetic fields in the two tori [37].

The difference between the D9-D5 and the D9-D7 systems is that in the first case the instanton size, equivalent to the D9-D5 hypermultiplet vev's, is a flat direction due to supersymmetry and the limit of small vev can be captured by an effective field theory description. For the D9-D7 system, due to the stringy value of the tachyonic mass, the process cannot be captured reliably by field theory. However, as we saw in section 4 , for the magnetized D9-brane description on the torus with minimum magnetic quantum number, there is a similar classical solution parametrized by a parameter $z_{0}$, which is similar to the instanton size of the D9-D5 brane system. Indeed, in the limit $z_{0} \rightarrow 0$, the solution describes a localized source and a localized vev for the negatively charged fields at the origin of the torus, which are the D7-branes. On the other hand, in the large $z_{0}$ limit, the solution becomes a constant magnetic field on the D9-branes, plus a profile for the tachyon which approaches a constant in the $z_{0} \gg R$ limit. This fits with the tachyon condensation in the D9-D7 system above. In section 4 , we constructed a classical solution for arbitrary flux that has a singular limit for $z_{0} \rightarrow 0$ corresponding to a localized flux, but we do not know the classical solution interpolating from this singular solution to a constant magnetic field. As we mentioned in section 4, such a solution could correspond to several localized sources in the singular limit.

Finally, tachyon condensation in the antisymmetric representation $(\mathbf{1}, \mathbf{d}(\mathbf{d}-\mathbf{1}) / \mathbf{2})$ breaks $\mathrm{U}(16)$ to $\mathrm{USp}(16)$. The final state is supersymmetric, so a natural question is the 

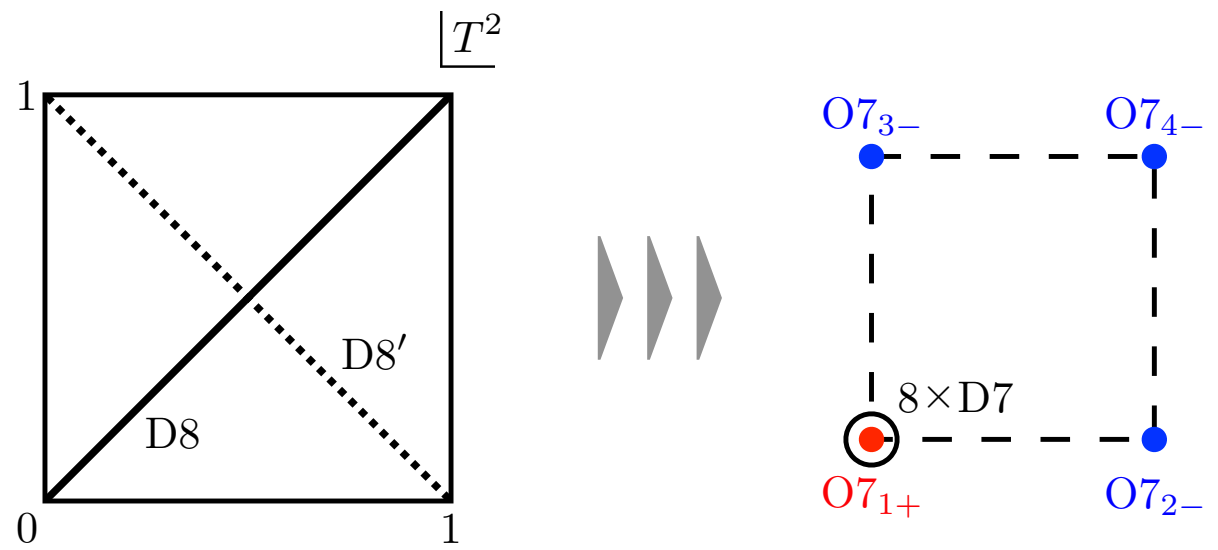

Figure 2. The left picture represents the type IIA orientifold with intersecting D8-branes. After tachyon condensation and one T-duality the conjectured final theory on the right is a type IIB orientifold with D7- and O7-planes where tachyon condensation changes one O7_-plane into an $\mathrm{O} 7_{+}$-plane.

string theory interpretation of it, if any. Fortunately, there is only one candidate superstring in $8 \mathrm{~d}$, which does have gauge group USp(16), to which we now turn.

\section{The $8 \mathrm{~d}$ USp(16) superstring as magnetized type I superstring after tachyon condensation}

To our knowledge there exists only one eight-dimensional superstring theory that can be the ground state after tachyon condensation. Its gauge group is USp(16), the group obtained after tachyon condensation as described in section 4 . This $8 \mathrm{~d}$ string theory was first constructed by Bianchi, Pradisi and Sagnotti [38, 39], and its geometry was understood later on by Witten using a T-dual picture [40]. The theory has also a dual description in terms of CHL strings [41, 42]. The original description $[38,39]$ makes use of the antisymmetric tensor field $B_{a b}$. This field is odd under world-sheet parity and therefore it is projected out by the orientifold projection $\Omega$ in type I superstring. However, this still leaves the possibility to add a quantized value $\frac{2}{\alpha^{\prime}} B_{a b} \in \mathbb{Z}$. The corresponding $8 \mathrm{~d}$ theory is supersymmetric and, in the absence of additional Wilson lines, it has gauge group USp(16) from the open string sector.

The T-dual version of the resulting theory has a particularly simple interpretation [40]. In the presence of the discrete background $B_{a b}$ T-duality in two coordinates acts as $R_{1} \rightarrow \frac{\alpha^{\prime}}{2 R_{1}}, R_{2} \rightarrow \frac{\alpha^{\prime}}{2 R_{2}}$. The T-dual of the standard $\mathrm{SO}(32)$ type I superstring turns the original O9_-plane wrapping the torus into four $\mathrm{O} 7$--planes at orientifold fixed points. On the contrary, the T-dual of the $\operatorname{USp}(16)$ superstring turns the original O9_-plane into three $\mathrm{O} 7_{-}$-planes and one $\mathrm{O} 7_{+}$-plane on the orientifold. While an $\mathrm{O} 7_{-}$-plane has charge (and tension) equal to -4 in units of a regular D7-brane charge, an $\mathrm{O} 7_{+}$-plane has charge (and tension) equal to +4 . The switch $\mathrm{O}_{-} \rightarrow \mathrm{O} 7_{+}$has the overall effect of halving the $\mathrm{RR}$ tadpole and therefore the rank of the gauge group, a fact that requires addition of only eight D7-branes. Furthermore, while D7-branes on top of an O7_-plane lead to an orthogonal (SO) gauge group, D7-branes on top of an $\mathrm{O} 7_{+}$-plane lead to a symplectic 
(USp) gauge group. Therefore, the configuration with all the D7-branes sitting on top of the $\mathrm{O} 7+$-plane leads to the gauge group $\operatorname{USp}(16)$. This configuration is depicted in the right part of figure 2 .

The open string spectrum is nicely encoded in the partition functions and it can be worked out using standard methods. We choose the type I string description with discrete value of $B_{a b}$, in which the cylinder and the Moebius amplitudes are given by

$$
\begin{aligned}
\mathcal{A} & =\frac{N^{2}}{2} \int_{0}^{\infty} \frac{d \tau_{2}}{\tau_{2}^{5}} \frac{V_{8}-S_{8}}{\eta^{8}}\left(P_{0}^{(1)}+P_{\frac{1}{2}}^{(1)}\right)\left(P_{0}^{(2)}+P_{\frac{1}{2}}^{(2)}\right) \\
\mathcal{M} & =\frac{N}{2} \int_{0}^{\infty} \frac{d \tau_{2}}{\tau_{2}^{5}} \frac{\hat{V}_{8}-\hat{S}_{8}}{\hat{\eta}^{8}}\left[\left(P_{0}^{(1)}-P_{\frac{1}{2}}^{(1)}\right) P_{0}^{(2)}-\left(P_{0}^{(1)}+P_{\frac{1}{2}}^{(1)}\right) P_{\frac{1}{2}}^{(2)}\right] .
\end{aligned}
$$

Here $P_{a}^{(i)}$ are the momentum sums

$$
P_{a}^{(i)}=\sum_{m_{i}} q^{\pi \alpha^{\prime}\left(m_{i}+a\right)^{2} / R_{i}^{2}}, \quad q=e^{2 \pi i \tau},
$$

where $R_{i}$ are the radii of the type I torus. Without the string excitations one obtains the open-string partition function

$$
\begin{aligned}
\mathcal{A}+\mathcal{M}=\int_{0}^{\infty} \frac{d \tau_{2}}{\tau_{2}^{5}} & \left(\frac{V_{8}-S_{8}}{\eta^{8}}\right)_{0}\left(\frac{N(N+1)}{2} P_{0}^{(1)} P_{0}^{(2)}\right. \\
& \left.+\frac{N(N-1)}{2}\left(P_{0}^{(1)} P_{\frac{1}{2}}^{(2)}+P_{\frac{1}{2}}^{(1)} P_{0}^{(2)}+P_{\frac{1}{2}}^{(1)} P_{\frac{1}{2}}^{(2)}\right)\right) .
\end{aligned}
$$

The $8 \mathrm{~d}$ spectrum is supersymmetric and corresponds to the gauge group $\operatorname{USp}(N)$, with $N=16$ fixed by the RR tadpole conditions. The field theory part of the spectrum can be read off from eq. (7.3). It contains massless states and several massive towers of the 2-torus:

- one massless gauge multiplet and KK towers in the symmetric (adjoint) representation of $\operatorname{USp}(16)$, with masses

$$
M_{1}^{2}=\frac{m_{1}^{2}}{R_{1}^{2}}+\frac{m_{2}^{2}}{R_{2}^{2}}
$$

- three massive gauge multiplets and KK towers in the antisymmetric representation of $\operatorname{USp}(16)$, with masses

$$
M_{2}^{2}=\frac{\left(m_{1}+\frac{1}{2}\right)^{2}}{R_{1}^{2}}+\frac{m_{2}^{2}}{R_{2}^{2}}, M_{3}^{2}=\frac{m_{1}^{2}}{R_{1}^{2}}+\frac{\left(m_{2}+\frac{1}{2}\right)^{2}}{R_{2}^{2}}, M_{4}^{2}=\frac{\left(m_{1}+\frac{1}{2}\right)^{2}}{R_{1}^{2}}+\frac{\left(m_{2}+\frac{1}{2}\right)^{2}}{R_{2}^{2}},
$$

where $R_{1}, R_{2}$ are the two radii of the type I torus. It is remarkable that this spectrum agrees with (5.16), the mass spectrum after tachyon condensation obtained in section 5 where, for simplicity, a square torus was chosen $\left(R_{1}=R_{2}=R\right)$.

The string vacuum just described is the only one preserving supersymmetry in $8 \mathrm{~d}$ with gauge group USp(16). It is therefore the only candidate for the endpoint of tachyon 
condensation in the magnetized U(16) 8d type I string. Hence, we are led to conjecture that the two string vacua are equivalent. However, since the rank of the gauge group is divided by two, the number of branes is halved in the process. In the T-dual picture, we need to understand the surprising connection between tachyon condensation and the conversion $\mathrm{O} 7_{-} \rightarrow \mathrm{O} 7_{+}$. While we do not have a full proof of the equivalence between the two string vacua, the following facts appear to support our conjecture.

Consider type I string with magnetized D9-branes wrapped on a torus $T^{2}$ with coordinates $y_{1}, y_{2}$, and a magnetic field along a Cartan generator commuting with $\mathrm{SU}(16)$. After T-duality on $y_{2}$, the magnetized D9-branes become a stack of D8-branes making an angle $\tan \theta=k R_{2}^{\prime} / R_{1}$ with the O8-planes, where $R_{2}^{\prime}$ is the T-dual radius. The tachyons in the antisymmetric representation of $\mathrm{U}(16)$ are stretched between the brane with wrapping numbers $(n, m)=(1, k)$ and its image $(1,-k) .{ }^{14}$ The corresponding wave functions are exponentially localized at their $I=2 k$ intersections, one at the origin and the others depending on $k$ (as an example, for $k=1$ there is a second intersection in the middle of the torus, see the left part of figure 2). Suppose the tachyon is condensing at the origin. Tachyon condensation corresponds to brane recombination. Here, the two cycles of the branes and their images recombine into a single factorizable cycle $(2,0)$, which is parallel to the cycle wrapped by the O8-plane at the origin $y_{2}=0$. The corresponding D8-branes have gauge group USp(16), as already discussed. The halving of the rank of the gauge group can be related to the resulting wrapping number $(2,0)$, which doubles the length of the recombined D8-branes. Since half of the D8-branes disappear in the process, presumably the nature of the O8-plane is also changed, in a way that cannot be captured in perturbation theory. ${ }^{15}$ If one now T-dualizes also the $y_{1}$-coordinate, one ends up with D7branes localized at the origin of the dual torus $\left(y_{1}, y_{2}\right)=(0,0)$, with gauge group USp(16). The same T-dualities for the $\mathrm{SO}(32)$ type I string (with no magnetic field) would of course lead to D7-branes with gauge group $\mathrm{SO}(32)$.

To shed some light on the conversion of one O7-plane, it is simpler to use the other, singular limit in which the magnetized D9-branes are described by the D9-D7 brane system, as described in section 6. In our case, we start with 16 non-BPS D7-branes in SO(32) type I string, with gauge group $\mathrm{U}(16)$. As described in the previous section, there are two complex tachyons in the spectrum, in the representations $(\mathbf{3 2}, \mathbf{1 6})$ and $(\mathbf{1}, \mathbf{1 2 0})$ of $\mathrm{SO}(32) \times \mathrm{U}(16)$. After two T-dualities in $y_{1}, y_{2}$, we obtain a non-BPS D9-brane with gauge group U(16) and a BPS D7-brane with gauge group $\mathrm{SO}(32)$, located at the origin of the torus. There are also the standard four $\mathrm{O}_{7}$-planes of the type IIB orientifold with the T-dual orientifold projection $\Omega^{\prime}=\Omega \Pi(-1)^{F}$, where $\Pi$ is the reflection $\left(y_{1}, y_{2}\right) \rightarrow\left(-y_{1},-y_{2}\right)$ and $(-1)^{F}$ is

\footnotetext{
${ }^{14}$ As well known from the intersecting brane picture, the magnetic field background can be generalized to arbitrary (coprime) wrapping numbers $m$ and $n$ [10]. If $n>1$, the resulting gauge group for one magnetized stack is $\mathrm{U}(16 / n)$ and is consistent if $16 / n$ is integer. The resulting effective field theory is more complicated since in addition to antisymmetric representations of the unitary gauge group, of multiplicity $m(n+1)$, there are also symmetric representations, of multiplicity $m(n-1)$. Both representations contain tachyonic scalars, which also make the tachyon condensation discussion more involved. This is a very interesting setup, which is however beyond the goals of our current paper.

${ }^{15}$ Taking into account the resulting gauge group USp(16), the torus in type IIA orientifold should become tilted, and the D8-branes and the O8-plane should acquire the wrapping numbers $(2,-1)[43,44]$.
} 
the spacetime fermion number. The condensation of the bi-fundamental tachyon $(\mathbf{3 2}, \mathbf{1 6})$ breaks the gauge group to the diagonal $\mathrm{U}(16)$. The condensation of the tachyon in the antisymmetric representation $(\mathbf{1}, \mathbf{1 2 0})$ breaks the gauge group to USp $(16)$ and halves the number of branes. What happens to the other eight branes, which disappear at the origin at the torus? Checking the charges/tension of these objects, one notices the suggestive equality

$$
Q_{\mathrm{O} 7_{+}}=Q_{\mathrm{O} 7_{-}}+8 Q_{\mathrm{D} 7} .
$$

This leads to the conjecture that the eight branes disappearing after tachyon condensation form a bound state with the coincident $\mathrm{O} 7$--plane, the resulting object having precisely the charge and tension of an $\mathrm{O} 7_{+}$-plane! The orientifold conversion can be compared to the $Q_{\mathrm{O} 3_{+}}=Q_{\mathrm{O} 3_{-}}+1 / 2 Q_{\mathrm{D} 3}$ needed in S-duality. While the $1 / 2$ stack (or rigid) D3 has no position moduli and, intuitively, can generate a bound state with the original O3-, in our case the 8 D7-branes probably form a bound state with the O7_-plane due to the tachyon condensation, and the corresponding open-string degrees of freedom become massive in the process. It would clearly be of great interest to find further evidence for this equivalence. However, this appears to be the only logical interpretation of the tachyon condensation in the context we discussed. Finally, notice that the results of section 3 exclude the interpretation of a USp(16) type I vacuum in terms of non-Abelian Wilson lines.

\section{Conclusions and open questions}

Understanding the vacuum structure of intersecting D-brane models and their T-dual magnetic compactifications is of crucial importance for potential phenomenological applications. In the interesting class of orientifold compactifications with chiral fermions, Wilson lines have generally been used to remove tachyons. Since quantum corrections have been shown to prevent local minima for Wilson lines, it is important to study tachyon condensation for vacua without Wilson lines.

The goal of this paper has been to analyze the simplest case of tachyon condensation which occurs in eight-dimensional type I string theory with magnetized branes wrapping a torus. This setup is T-dual to type IIA orientifolds with intersecting D8-branes. We have analyzed in detail the simplest case where in the type IIA description all 16 D8-branes are rotated by the same angle, leading to the gauge group $\mathrm{U}(16)$ in the open string spectrum. In this case a tachyon occurs at the intersection of the D8-branes with their images. We have shown that after tachyon condensation the resulting gauge group becomes USp(16). By analyzing the field theory condensation process, we have argued that the final string theory should be equivalent to the unique known USp(16) superstring in eight dimensions. The latter has the peculiar feature that, in a T-dual version with D7- and O7-planes, one orientifold plane is of exotic type $\mathrm{O} 7_{+}$, whereas the three others are standard $\mathrm{O} 7_{-}$-planes. We argue that tachyon condensation induces dynamically an $\mathrm{O} 7_{-} \rightarrow \mathrm{O} 7_{+}$conversion, by generating a bound state of the $\mathrm{O} 7$--plane with half of the D7-branes that naively disappear in the condensation process. The result is supported by various arguments and in particular by the equality

$$
Q_{\mathrm{O} 7_{+}}=Q_{\mathrm{O} 7_{-}}+8 Q_{\mathrm{D} 7},
$$

yielding a consistent picture of a type IIB orientifold. 
The stable vacuum after tachyon condensation is supersymmetric and has no chiral fermions. It remains a challenge to find intersecting D-brane models with broken supersymmetry and chiral fermions.

\section{Acknowledgments}

We thank Costas Bachas, Markus Dierigl, Jihad Mourad, Augusto Sagnotti and Timo Weigand for valuable discussions and correspondence. E.D. was supported in part by the "Agence Nationale de la Recherche" (ANR). Y.T. is supported in part by Grants-in-Aid for JSPS Overseas Research Fellow (No. 18J60383) from the Ministry of Education, Culture, Sports, Science and Technology in Japan, and in part by Scuola Normale, by INFN (IS GSS-Pi) and by the MIUR-PRIN contract 2017CC72MK_003.

\section{A Flux quantization}

The torus $T^{2}$ is not simply connected so that vector fields and matter fields are represented by fibre bundles. The case of a monopole field on a sphere with constant flux density has been thoroughly discussed in ref. [45], and homogeneous magnetic fields on a torus have been considered in refs. $[9,32]$. In these papers the vector potential for constant magnetic flux was chosen in Landau gauge so that the torus could be covered with two patches. Another convenient choice is the symmetric gauge [33] which requires four patches, for instance the ones shown in figure 3 . Here the vector potential is given by

$$
\begin{aligned}
& \text { (a) } \quad A_{1}=-\frac{f}{2} y_{2}, \quad A_{2}=\frac{f}{2} y_{1}, \quad 0 \leq y_{1}<\frac{1}{2}, \quad 0 \leq y_{2}<\frac{1}{2} \\
& \text { (b) } \quad A_{1}=-\frac{f}{2}\left(y_{2}-1\right), \quad A_{2}=\frac{f}{2} y_{1}, \quad 0 \leq y_{1}<\frac{1}{2}, \quad \frac{1}{2} \leq y_{2}<1 \\
& \text { (c) } \quad A_{1}=-\frac{f}{2} y_{2}, \quad A_{2}=\frac{f}{2}\left(y_{1}-1\right), \quad \frac{1}{2} \leq y_{1}<1, \quad 0 \leq y_{2}<\frac{1}{2} \\
& \text { (d) } \quad A_{1}=-\frac{f}{2}\left(y_{2}-1\right), \quad A_{2}=\frac{f}{2}\left(y_{1}-1\right), \quad \frac{1}{2} \leq y_{1}<1, \quad \frac{1}{2} \leq y_{2}<1 \text {, }
\end{aligned}
$$

leading to the constant magnetic field $F=d A=f v$, with $v=d y_{1} \wedge d y_{2}$. Each patch overlaps with two other patches at two boundaries each, see figure 3 .

The transition function that relates fields at the same point in the two patches is given by $[45]$

$$
\begin{aligned}
\phi_{f} & =S_{f e} \phi_{e}, \quad S_{f e}=e^{i \Lambda_{f e}}, \quad S_{f e}=S_{e f}^{-1} \\
A_{m}^{f} & =A_{m}^{e}+\frac{i}{g} S_{f e}^{-1} \partial_{m} S_{f e}=A_{m}^{e}-\frac{1}{g} \partial_{m} \Lambda_{f e} .
\end{aligned}
$$

Given the vector fields (A.1) in the patches $(a),(b),(c)$ and $(d)$ this yields the transition functions

$$
\Lambda_{b a}\left(y_{1}, 1 / 2\right)=\Lambda_{d c}\left(y_{1}, 1 / 2\right)=-\frac{g f}{2} y_{1}, \quad \Lambda_{c a}\left(1 / 2, y_{2}\right)=\Lambda_{d b}\left(1 / 2, y_{2}\right)=\frac{g f}{2} y_{2} .
$$




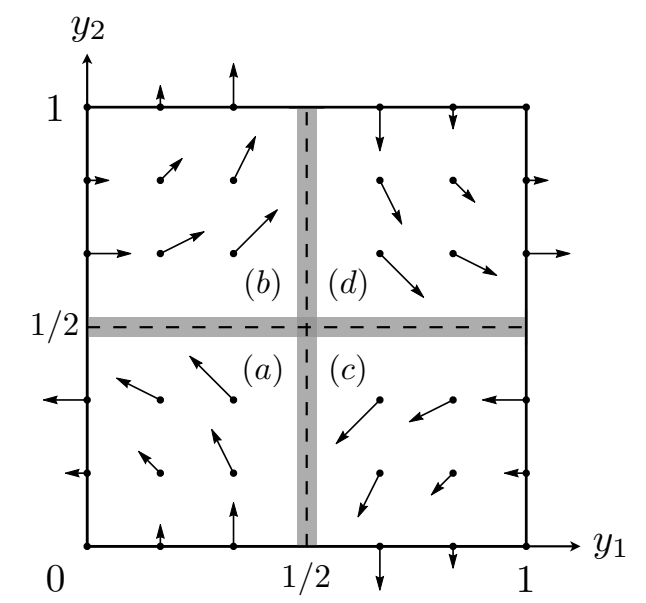

Figure 3. Vector potential for constant magnetic flux on a torus with four patches.

The consistency condition at the overlap of all four patches,

$$
\Lambda_{a c}(1 / 2,1 / 2) \Lambda_{c d}(1 / 2,1 / 2) \Lambda_{d b}(1 / 2,1 / 2) \Lambda_{b a}(1 / 2,1 / 2)=e^{-i g f}=1,
$$

implies flux quantization, $g f=2 \pi M, M \in \mathbb{Z}$. The transition functions at the boundaries of the fundamental domain of the torus are trivial.

Starting at $y_{2}=1 / 2$ in patch $(b)$ and going around the torus in $y_{2}$-direction via patch (b) and patch $(a)$ until $y_{2}=1 / 2$ in patch $(a)$, the vector field changes from $A_{1}=f / 4$ to $A_{1}=-f / 4$. This necessitates a non-trivial transition function $S_{b a}$, which in the literature on magnetized tori usually occurs as twisted boundary condition (see, for example [33]),

$$
\phi\left(y_{1}, y_{2}+1\right)=S_{b a}^{-1}\left(y_{1}, 1 / 2\right) \phi\left(y_{1}, y_{2}\right)=e^{i q f y_{1} / 2} \phi\left(y_{1}, y_{2}\right) .
$$

Analogously, one obtains for a lattice translation in $y_{1}$-direction,

$$
\phi\left(y_{1}+1, y_{2}\right)=S_{c a}^{-1}\left(1 / 2, y_{2}\right) \phi\left(y_{1}, y_{2}\right)=e^{-i q f y_{2} / 2} \phi\left(y_{1}, y_{2}\right) .
$$

This means that the twist factors are given by the transition functions on the torus in $y_{1^{-}}$ and $y_{2}$-direction, respectively.

Open Access. This article is distributed under the terms of the Creative Commons Attribution License (CC-BY 4.0), which permits any use, distribution and reproduction in any medium, provided the original author(s) and source are credited.

\section{References}

[1] L.E. Ibáñez and A.M. Uranga, String theory and particle physics: An introduction to string phenomenology, Cambridge University Press (2012) [InSPIRE].

[2] C. Angelantonj and A. Sagnotti, Open strings, Phys. Rept. 371 (2002) 1 [Erratum ibid. 376 (2003) 407] [hep-th/0204089] [INSPIRE]. 
[3] R. Blumenhagen, B. Körs, D. Lüst and S. Stieberger, Four-dimensional String Compactifications with D-branes, Orientifolds and Fluxes, Phys. Rept. 445 (2007) 1 [hep-th/0610327] [INSPIRE].

[4] N. Arkani-Hamed and S. Dimopoulos, Supersymmetric unification without low energy supersymmetry and signatures for fine-tuning at the LHC, JHEP 06 (2005) 073 [hep-th/0405159] [INSPIRE].

[5] G.F. Giudice and A. Romanino, Split supersymmetry, Nucl. Phys. B 699 (2004) 65 [Erratum ibid. 706 (2005) 487] [hep-ph/0406088] [INSPIRE].

[6] I. Antoniadis and S. Dimopoulos, Splitting supersymmetry in string theory, Nucl. Phys. B 715 (2005) 120 [hep-th/0411032] [INSPIRE].

[7] W. Buchmüller, M. Dierigl, F. Ruehle and J. Schweizer, Split symmetries, Phys. Lett. B $\mathbf{7 5 0}$ (2015) 615 [arXiv : 1507.06819] [inSPIRE].

[8] C. Bachas, A Way to break supersymmetry, hep-th/9503030 [INSPIRE].

[9] A. Abouelsaood, C.G. Callan Jr., C.R. Nappi and S.A. Yost, Open Strings in Background Gauge Fields, Nucl. Phys. B 280 (1987) 599 [INSPIRE].

[10] R. Blumenhagen, L. Görlich, B. Körs and D. Lüst, Noncommutative compactifications of type-I strings on tori with magnetic background flux, JHEP 10 (2000) 006 [hep-th/0007024] [INSPIRE].

[11] C. Angelantonj, I. Antoniadis, E. Dudas and A. Sagnotti, Type I strings on magnetized orbifolds and brane transmutation, Phys. Lett. B 489 (2000) 223 [hep-th/0007090] [INSPIRE].

[12] M. Berkooz, M.R. Douglas and R.G. Leigh, Branes intersecting at angles, Nucl. Phys. B 480 (1996) 265 [hep-th/9606139] [INSPIRE].

[13] G. Aldazabal, S. Franco, L.E. Ibáñez, R. Rabadán and A.M. Uranga, $D=4$ chiral string compactifications from intersecting branes, J. Math. Phys. 42 (2001) 3103 [hep-th/0011073] [INSPIRE].

[14] G. Aldazabal, S. Franco, L.E. Ibáñez, R. Rabadán and A.M. Uranga, Intersecting brane worlds, JHEP 02 (2001) 047 [hep-ph/0011132] [INSPIRE].

[15] L.E. Ibáñez, F. Marchesano and R. Rabadán, Getting just the standard model at intersecting branes, JHEP 11 (2001) 002 [hep-th/0105155] [INSPIRE].

[16] R. Blumenhagen, B. Körs, D. Lüst and T. Ott, The standard model from stable intersecting brane world orbifolds, Nucl. Phys. B 616 (2001) 3 [hep-th/0107138] [INSPIRE].

[17] M. Cvetič, G. Shiu and A.M. Uranga, Chiral four-dimensional $N=1$ supersymmetric type $2 A$ orientifolds from intersecting D6 branes, Nucl. Phys. B 615 (2001) 3 [hep-th/0107166] [INSPIRE].

[18] W. Buchmüller, E. Dudas and Y. Tatsuta, Quantum corrections for D-brane models with broken supersymmetry, JHEP 12 (2019) 022 [arXiv:1909.03007] [INSPIRE].

[19] I. Antoniadis, E. Gava, K.S. Narain and T.R. Taylor, Duality in superstring compactifications with magnetic field backgrounds, Nucl. Phys. B 511 (1998) 611 [hep-th/9708075] [INSPIRE].

[20] K. Hashimoto and S. Nagaoka, Recombination of intersecting D-branes by local tachyon condensation, JHEP 06 (2003) 034 [hep-th/0303204] [INSPIRE]. 
[21] F.T.J. Epple and D. Lüst, Tachyon condensation for intersecting branes at small and large angles, Fortsch. Phys. 52 (2004) 367 [hep-th/0311182] [INSPIRE].

[22] A. Sen, Tachyon dynamics in open string theory, Int. J. Mod. Phys. A 20 (2005) 5513 [hep-th/0410103] [INSPIRE].

[23] M. Bianchi and A. Sagnotti, On the systematics of open string theories, Phys. Lett. B 247 (1990) 517 [INSPIRE].

[24] M. Bianchi and A. Sagnotti, Twist symmetry and open string Wilson lines, Nucl. Phys. B 361 (1991) 519 [INSPIRE].

[25] N. Marcus, A. Sagnotti and W. Siegel, Ten-dimensional Supersymmetric Yang-Mills Theory in Terms of Four-dimensional Superfields, Nucl. Phys. B 224 (1983) 159 [INSPIRE].

[26] N. Arkani-Hamed, T. Gregoire and J.G. Wacker, Higher dimensional supersymmetry in 4-D superspace, JHEP 03 (2002) 055 [hep-th/0101233] [INSPIRE].

[27] J. Wess and J. Bagger, Supersymmetry and supergravity, Princeton University Press, Princeton, NJ, U.S.A. (1992).

[28] W. Buchmüller, M. Dierigl and E. Dudas, Flux compactifications and naturalness, JHEP 08 (2018) 151 [arXiv: 1804.07497] [INSPIRE].

[29] L.-F. Li, Group Theory of the Spontaneously Broken Gauge Symmetries, Phys. Rev. D 9 (1974) 1723 [INSPIRE].

[30] H. Georgi, Lie algebras in particle physics. From isospin to unified theories, Front. Phys. 54 (1982) 1 [INSPIRE].

[31] H.M. Lee, H.P. Nilles and M. Zucker, Spontaneous localization of bulk fields: The Six-dimensional case, Nucl. Phys. B 680 (2004) 177 [hep-th/0309195] [INSPIRE].

[32] W. Buchmüller, M. Dierigl and Y. Tatsuta, Magnetized orbifolds and localized flux, Annals Phys. 401 (2019) 91 [arXiv:1810.06362] [INSPIRE].

[33] D. Cremades, L.E. Ibáñez and F. Marchesano, Computing Yukawa couplings from magnetized extra dimensions, JHEP 05 (2004) 079 [hep-th/0404229] [INSPIRE].

[34] E. Dudas, J. Mourad and A. Sagnotti, Charged and uncharged D-branes in various string theories, Nucl. Phys. B 620 (2002) 109 [hep-th/0107081] [InSPIRE].

[35] E. Witten, Small instantons in string theory, Nucl. Phys. B 460 (1996) 541 [hep-th/9511030] [INSPIRE].

[36] M.R. Douglas, Branes within branes, NATO Sci. Ser. C 520 (1999) 267 [hep-th/9512077] [INSPIRE].

[37] C. Angelantonj, C. Condeescu, E. Dudas and G. Pradisi, Non-perturbative transitions among intersecting-brane vacua, JHEP 07 (2011) 123 [arXiv: 1105.3465] [INSPIRE].

[38] M. Bianchi, G. Pradisi and A. Sagnotti, Toroidal compactification and symmetry breaking in open string theories, Nucl. Phys. B 376 (1992) 365 [InSPIRE].

[39] M. Bianchi, A note on toroidal compactifications of the type-I superstring and other superstring vacuum configurations with sixteen supercharges, Nucl. Phys. B 528 (1998) 73 [hep-th/9711201] [INSPIRE].

[40] E. Witten, Toroidal compactification without vector structure, JHEP 02 (1998) 006 [hep-th/9712028] [INSPIRE]. 
[41] S. Chaudhuri, G. Hockney and J.D. Lykken, Maximally supersymmetric string theories in $D<10$, Phys. Rev. Lett. 75 (1995) 2264 [hep-th/9505054] [INSPIRE].

[42] M. Bianchi, E. Gava, J.F. Morales and K.S. Narain, D strings in unconventional type-I vacuum configurations, Nucl. Phys. B 547 (1999) 96 [hep-th/9811013] [INSPIRE].

[43] R. Blumenhagen, B. Körs and D. Lüst, Type I strings with F flux and B flux, JHEP 02 (2001) 030 [hep-th/0012156] [INSPIRE].

[44] C. Bachas, M. Bianchi, R. Blumenhagen, D. Lüst and T. Weigand, Comments on Orientifolds without Vector Structure, JHEP 08 (2008) 016 [arXiv:0805.3696] [INSPIRE].

[45] T.T. Wu and C.N. Yang, Concept of Nonintegrable Phase Factors and Global Formulation of Gauge Fields, Phys. Rev. D 12 (1975) 3845 [INSPIRE]. 